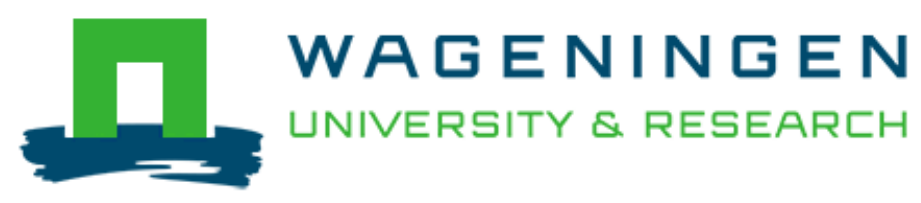

Modelling ultrafiltration performance by integrating local (critical) fluxes along the membrane length

Aguirre-Montesdeoca, V., Janssen, A. E. M., Van der Padt, A., \& Boom, R. M.

This is a "Post-Print" accepted manuscript, which has been published in "Journal of Membrane Science"

This version is distributed under a non-commercial no derivatives Creative Commons

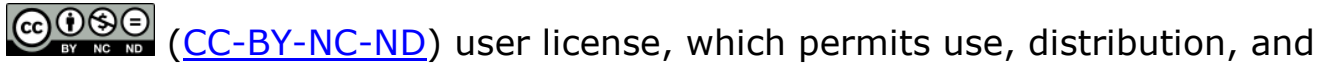
reproduction in any medium, provided the original work is properly cited and not used for commercial purposes. Further, the restriction applies that if you remix, transform, or build upon the material, you may not distribute the modified material.

Please cite this publication as follows:

Aguirre-Montesdeoca, V., Janssen, A. E. M., Van der Padt, A., \& Boom, R. M. (2019). Modelling ultrafiltration performance by integrating local (critical) fluxes along the membrane length. Journal of Membrane Science, 578, 111-125. DOI:

10.1016/j.memsci.2019.02.040

You can download the published version at:

https://doi.org/10.1016/j.memsci.2019.02.040 


\title{
Modelling ultrafiltration performance by integrating local (critical) fluxes along the membrane length.
}

\author{
Victor Aguirre-Montesdeoca*1,2, Anja E. M. Janssen², A. Van der Padt ${ }^{2,3}$, and R. M. Boom ${ }^{2}$ \\ ${ }^{1}$ Institute for Sustainable Process Technology, P.O. Box 247, 3800 AE, Amersfoort, The Netherlands. \\ ${ }^{2}$ Wageningen University, Food Process Engineering Group, P.O. Box 17, 6700 AA, Wageningen, The \\ Netherlands. \\ ${ }^{3}$ FrieslandCampina, P.O. Box 1551, 3800 BN, Amersfoort, The Netherlands. \\ *Corresponding Author: E-mail: victor.aguirremontesdeoca@wur.nl
}

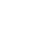

\section{ABSTRACT}

Despite the vast number of studies on the understanding and estimation of the permeate flux in ultrafiltration, most of them base their estimations on either one or another mechanism, without pointing out a clear 'bridge' between them. The aim of this paper is to assess these mechanisms on the determination of the permeate flux, using as feed a multicomponent mixture of $\mathrm{BSA}, \mathrm{NaCl}$ and $\mathrm{H}_{2} \mathrm{O}$.

Maxwell-Stefan Equations expressed as function of the components' volume fractions were used for an easier consideration of the non-idealities of the system. These non-idealities (hydration, adsorption, electrical interactions and volume exclusion) were critical in the local fluxes calculation, for which an increase in the thickness of the boundary layer along the filtration channel was considered.

The developed model proved to be suitable for the estimation of fluxes lower than the limiting flux. Since the non-idealities of the system can be calculated along the concentration polarization layer, no extra information on the protein diffusivity was needed. Additionally, the fact that the model includes all the components from the solution offers the possibility of including the rejection of the accompanying ions in the calculations.

Keywords: Local critical flux; gel layer; non-idealities; Maxwell-Stefan; protein ultrafiltration. 


\section{INTRODUCTION}

The ultrafiltration (UF) of proteins is an important process in the food and biotechnology industry, it is generally used for purification and concentration purposes, in which the retentate (concentrate) is usually the stream with highest value. The main drawback of this process is the marked flux decline obtained over time. This decrease can be larger than one order of magnitude depending on the process conditions and protein concentration [1-4]. It has, therefore, been subject of intensive study over the last five decades and many models and theories have been developed to explain the mechanisms that lead to this flux decrease.

The most popular theory that explains this flux decrease during UF is the so-called gel layer model . It assumes that the surface of the membrane is completely covered by a layer of proteins, which have reached their maximum concentration and, as consequence, formed a gel $[5,6]$. The gel increases the resistance of the membrane, lowering the permeate flux over time until it reaches a plateau.

Additionally, the effect of the osmotic pressure in the system counteracts the TMP, resulting in a lower driving force for convection. In theory, the permeate flux at steady state can be calculated with a balance between convection and dispersive forces (back diffusion). Nevertheless, Cohen and Probstein already in 1986 found out that the measured flux was much higher than the 'theoretical' one obtained with the aforementioned balance [7]. They speculated that the factor responsible for this effect might be related with the surface interaction between colloidal particles, a phenomenon related to the inherent charge of the macromolecules. In order to predict the steady state flux of the system, other studies have considered phenomena such as: purely osmotic pressure effects $[5,8]$, shear induced diffusion [9], inertial migration [10], DLVO theory [11], etc. In 1995 Bowen and Jenner even developed a rigorous dynamic model that accounted for many types of long and short range interaction between charged colloids[12]. Likewise, Jönsson and Jönsson worked out a model to perform the force balance in the concentration polarization layer, in which they included non-idealities due to high concentrations [13]. 
Most of the studies tend to base their predictions on either one or another model, without pointing out a clear integration between the different mechanisms to create a coherent mechanistic picture of the process. It has already been shown in UF applications that the gel does not grow uniformly on top of the membrane. Due to varying boundary layer thickness, the gel first appears at the outlet of the filtration channel and then it grows towards the inlet $[9,14]$. This means that under some conditions the membrane can be partially fouled and more than one mechanism can determine the resulting overall permeate flux as shown in Figure 1.

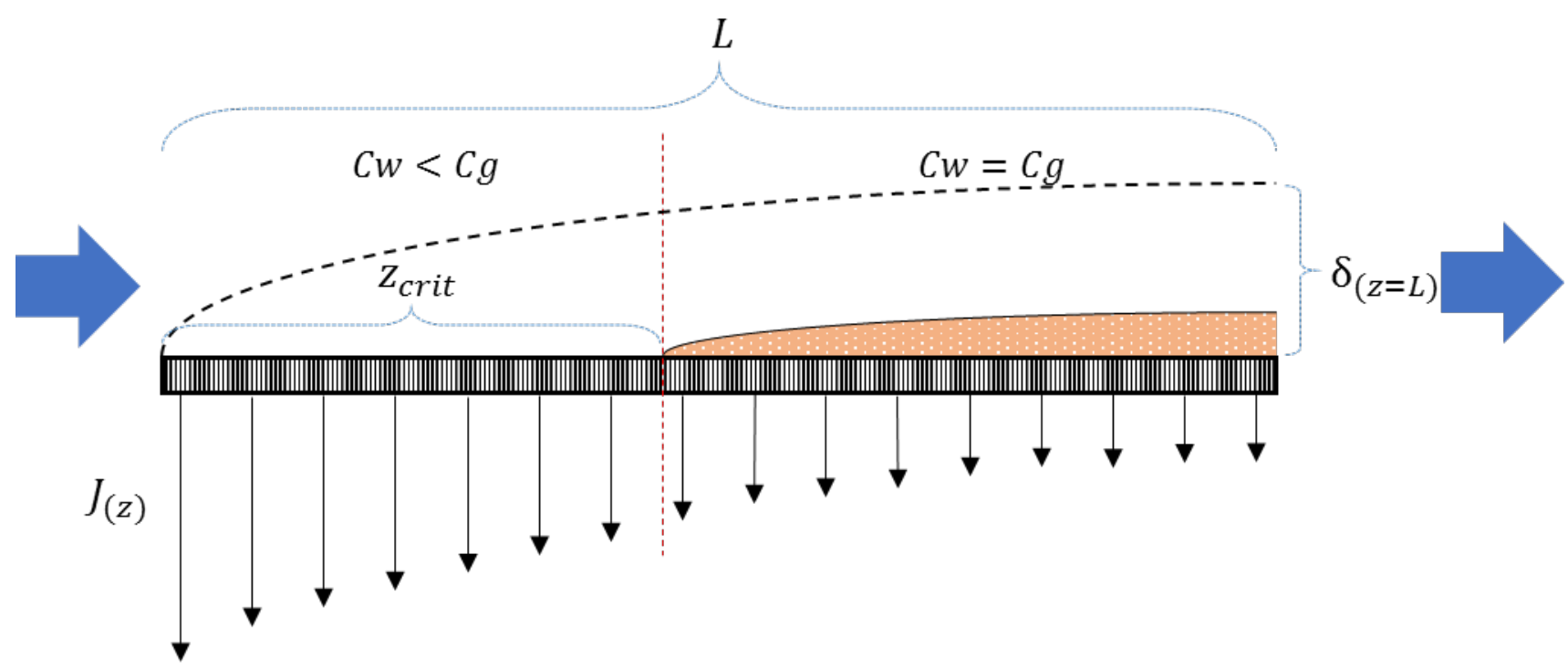

Figure 1. Representation of a flat membrane partially covered with gel and the resulting local fluxes $J_{(z)} . C_{w}$ stands for concentration of the protein at the membrane surface and $C_{g}$ is the gel concentration. $\delta$ represents the growing boundary layer along the filtration channel of length $L . z_{\text {crit }}$ is the distance from the inlet to the point where gel deposit appears.

The aim of this paper is to assess the mechanisms that determine the permeate flux in the ultrafiltration of protein solutions. Special attention is given to the effects of the electrical interactions between proteins and ions in the mixture. The model system chosen for this study was $\mathrm{BSA}, \mathrm{NaCl}$ and water. We used a long filtration channel (length $1 \mathrm{~m}$ ) to represent an actual filtration system, in which, due to a growing boundary layer down the channel, several flux limiting mechanisms may occur. To facilitate the modelling of the system, we chose a membrane that completely rejects BSA but let the 
1 accompanying ions pass freely. The experimental results are compared with the predictions of a

2 rigorous model built within the framework of the Maxwell-Stefan (M-S) equations.

\section{2. THEORETICAL ASPECTS}

4

5 Before starting the analysis of the mechanisms behind flux reduction in UF, it is necessary to mention

6 the definition of limiting and critical flux. The limiting flux is the maximum steady state permeate flux

7 that can be achieved by increasing TMP in the system [6]. Figure 2 shows that at the limiting flux,

8 increasing the pressure does not increase the steady state flux. It is generally accepted that at these

\subsection{Local critical fluxes}

conditions the membrane is totally fouled and pressure increments lead to thickening of the gel layer at the membrane surface, resulting in the same steady state flux. The critical flux, on the other hand, is the flux below which no fouling occurs; in other words, it is the flux required to overcome particle repulsion; exceeding this flux leads to the coagulation of the protein on the surface $[14,15]$. 


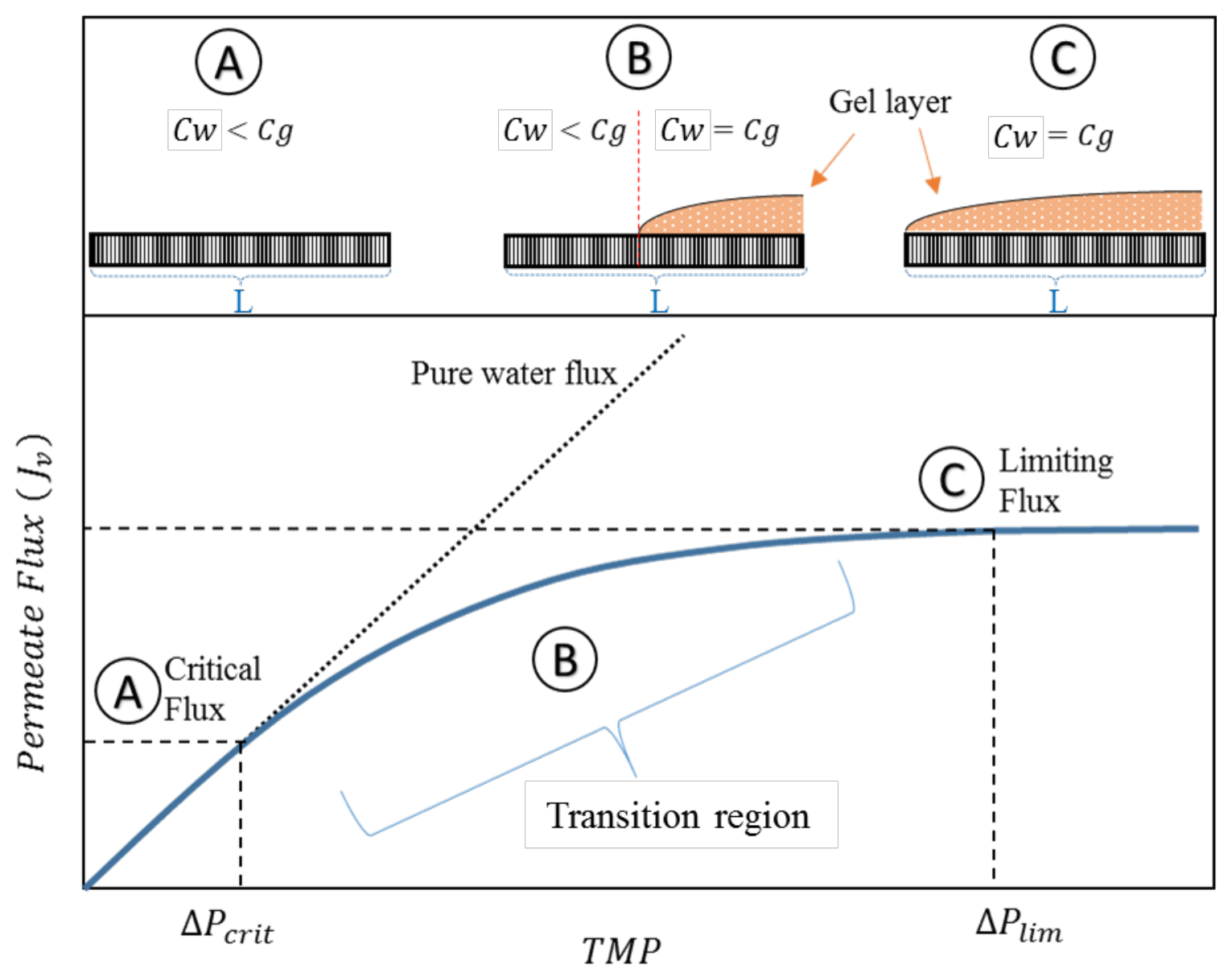

Figure 2. Permeate flux as function of transmembrane pressure (TMP) for the ultrafiltration of proteins. Region A represent membrane conditions below the critical flux, region B is the 'transition region' where the membrane is partially fouled, and region $\mathrm{C}$ represents the limiting conditions.

5 Bacchin made an interesting connection between the limiting and critical concepts when they introduced the concept of 'local critical fluxes'[15]. As shown in Figure 1, he described a system with a growing boundary layer along the membrane length, in which fouling occurs first in the outlet and extends towards the module inlet depending on the TMP of the system. In this way, critical fluxes depend on the position along the membrane, being lower at the outlet of the module than at the entrance. When working at sub-critical conditions, with a pressure lower than $\Delta P_{\text {crit }}$, the membrane remains clean without the formation of gel layer (Figure 2 region A). As the pressure increases, fouling starts appearing at the outlet of the membrane, showing that the critical flux has been exceeded. As the gel layer grows along the membrane, partially covering it, a non-linear dependency of the flux with respect to the pressure occurs (region B - Transition region). Finally, when the gel 
1 independent of the pressure (region C). In this study, variables like TMP and cross-flow velocity are

2 considered constant along the membrane due to the relatively large height of the channel.

3 It has been demonstrated that the concentration polarization in filtration systems can be represented using an stagnant film model, in which mass transfer is assumed to occur across a stagnant film of thickness $\delta$ [16]. In theory, local fluxes can be calculated as long as the local values for $\delta$ are known. Considering that a typical boundary layer for a laminar and developed flow follows a power law of $1 / 3$, the next relation can be used in agreement with Davis and Sherwood [9]:

$$
\delta_{(z)}=\delta_{(z=L)}\left(\frac{z}{L}\right)^{1 / 3}
$$

in which $z$ represents the position along the channel and $L$ is its total length. The calculation of the mean flux averaged over the membrane $\left(J_{v}\right)$ for a system totally covered by protein gel can be done by integrating the local fluxes as shown in Eq. 2, where $J_{\text {crit }}(z)$ represent the local critical flux at every position $\mathrm{z}[15]$.

$$
J_{v}=\frac{1}{L} \int_{0}^{L} J_{c r i t}(z) d z
$$

When the TMP is higher than the pressure needed to reach the limiting flux $\left(\Delta P_{\text {lim }}\right)$, the permeate flux is totally determined by the gel layer mechanism and Eq. 2 can be used. However, if $T M P<\Delta P_{\text {lim }}$ it could be that the membrane is partially fouled (region B in Figure 2) or not fouled at all (region A). In those cases, osmotic pressure influences the overall permeate flux. Therefore, if only a section of the membrane is fouled, more than one flux limiting mechanism is active. Therefore, both sections of the membrane should be analysed separately as shown in Eq. 3, in which $z_{\text {crit }}$ is the distance from the inlet of the channel to the point at which the gel first appears (see Figure 1) [15].

$$
J_{v}=\frac{1}{L}\left(\int_{0}^{z_{\text {crit }}} J_{\text {osmo }}(z) d z+\int_{z_{\text {crit }}}^{L} J_{\text {crit }}(z) d z\right)
$$

The calculation of the local fluxes with and without the presence of a gel layer $\left(J_{\text {crit }}\right.$ and $\left.J_{\text {osmo }}\right)$ needs to be performed considering all the driving forces and all the components of the system. Likewise, the 
thermodynamic non-idealities need to be included in the analysis. Therefore, the M-S equations can be used to combine the aforementioned aspects in one single model.

\subsection{Maxwell Stefan equations}

During UF, the concentration of the proteins increases in the concentration polarization layer since they are retained by the membrane. This concentration profile, together with that of the ions determine a gradient in the electrochemical potential, which together with the chemical potential are the driving forces of the system. At steady state, the forces acting on the solutes balance out in the concentration polarization layer. In a non-ideal multicomponent system, the most appropriate approach to describe the forces in this layer is using the M-S equations. These equations are can be envisaged as a force balance between the driving forces and the friction forces in the system [17].

A convenient way to present the M-S equations is shown in Eq. 4, in which the force balance at steady state in the concentration polarization layer is represented for molecule $i$. The molecular diffusion in this layer can be represented by a set of $m-1$ equations, being $m$ the number of components (including water as component $m$ ). The term at the left hand side represents the driving forces for solute $i$ and the one at the right represents the friction forces working over solute $i$. It is important to realize that the driving forces together are expressed as the electrochemical potential gradient $\left(\nabla \tilde{\mu}_{i}\right) . x$ represents the solutes mole fraction and $u$ are their linear velocities. $\bigoplus_{i j}$ is the M-S cross diffusion coefficient between species $i$ and $j[17,18]$.

$$
-\frac{x_{i}}{R T} \nabla_{T, P} \tilde{\mu}_{i}=\sum_{\substack{j=1 \\ j \neq i}}^{m} \frac{\left(u_{i}-u_{j}\right) x_{i} x_{j}}{\bigoplus_{i j}}
$$

The electrochemical potential gradient can be split into the chemical $\left(\nabla \mu_{i}\right)$ and the electrical potential gradients $(\nabla \Psi)$ [17]. Furthermore, it is convenient to express the equations in terms of volume fractions $(\varphi)$ instead of molar fractions because, as it is explained further in section 2.3, most of the non-idealities are functions of $\varphi$. Thus, considering: 


$$
x_{i}=\frac{\varphi_{i}}{\bar{V}_{i} C_{T}}
$$

1 in which $\bar{V}_{i}$ is the molar volume of $i$ and $C_{T}$ is the total molar concentration, we can obtain

$$
-\frac{\varphi_{i}}{\bar{V}_{i} C_{T} R T} \nabla \mu_{i}-\frac{\varphi_{i}}{\bar{V}_{i} C_{T}} \frac{Z_{i} F}{R T} \nabla \Psi=\sum_{\substack{j=1 \\ j \neq i}}^{m} \frac{\left(u_{i}-u_{j}\right)}{\bigoplus_{i j}} \frac{\varphi_{i}}{\bar{V}_{i} C_{T}} \frac{\varphi_{j}}{\bar{V}_{j} C_{T}}
$$

2 in which $Z_{i}$ is the charge of species $i$ and $F$ is the Faraday constant. Similarly, volume fluxes $\left(J_{i}\right)$ and

3 modified M-S diffusion coefficients can be defined using the following relations:

$$
\begin{aligned}
& J_{i}=\varphi_{i} u_{i} \\
& \bigoplus_{i j}^{V}=\bigoplus_{i j} \bar{V}_{j} C_{T}
\end{aligned}
$$

4 Here, it is important to mention that under this definition the modified M-S diffusion coefficients obey

5 the following symmetry in agreement with Onsager's reciprocal relations $[19,20]$ :

6

$$
\bigoplus_{i j}=\frac{\bigoplus_{i j}^{V}}{\bar{V}_{j} C_{T}}=\bigoplus_{j i}=\frac{\bigoplus_{j i}^{V}}{\bar{V}_{i} C_{T}} ; \quad \frac{\bigoplus_{i j}^{V}}{\bar{V}_{j}}=\frac{\bigoplus_{j i}^{V}}{\bar{V}_{i}}
$$

7 By combining Eqs.6-8, many variables cancel out, resulting in a simpler relation $[19,20]$ :

$$
\frac{\varphi_{i}}{R T} \nabla \mu_{i}+\frac{\varphi_{i} Z_{i} F}{R T} \nabla \Psi=\sum_{\substack{j=1 \\ j \neq i}}^{m} \frac{\left(J_{j} \varphi_{i}-J_{i} \varphi_{j}\right)}{\bigoplus_{i j}^{V}}
$$

8 If we consider that in UF the permeate flux is mostly water $\left(J_{v} \approx J_{m}\right)$, and the volume fraction of

9 water in the permeate is almost $1\left(\varphi_{m, p} \approx 1\right) . J_{i}$ for the ions can be related with $J_{v}$ as shown in Eq 10.

10 This equation let us link the fluxes with the concentrations in our system; it is known as 'bootstrap'

11 [18].

$12 \quad \frac{J_{i}}{J_{m}}=\frac{\varphi_{i, p}}{\varphi_{m, p}}$

$$
J_{i}=J_{v} \varphi_{i, p}
$$


1 For a system with 4 components: $\mathrm{BSA}(1), \mathrm{Na}^{+}(2), \mathrm{Cl}^{-}(3)$ and $\mathrm{H}_{2} \mathrm{O}(4)$, three equations equivalent to

2 Eq.9 corresponding to component 1,2 and 3 are needed. Additionally, the following condition is

3 necessary to calculate the volume fractions for component 4 :

$$
\sum_{i=1}^{m} \varphi_{i}=1
$$

4 In this study, the maximum volume fraction for BSA is considered to be $\varphi_{\text {gel }}=0.55[12,21]$.

5 Although it is expected that this value changes depending on $\mathrm{pH}$ and I, not enough experimental

6 information was found in literature. Nevertheless, this slight variation does not influence greatly the

7 overall results of the model.

8

\subsection{Non-idealities}

Several types of interactions occur between the components of the mixture. A summary is presented in Table 1. The relevance of these interactions is generally dependent on the concentration of the involved components in the mixture. Since the concentration of BSA increases greatly in the concentration polarization layer, most of the interactions become relevant and need to be considered to account for the thermodynamic non-ideality of the solution.

Table 1. Interactions between components of the feed mixture

\begin{tabular}{c|c|c|c|c}
\hline & BSA & Na+ & Cl- & H2O \\
\hline BSA & Excluded volume & Electric coupling & $\begin{array}{c}\text { Electric coupling } \\
\text { Cl- ads. to BSA }\end{array}$ & Hydration \\
\hline $\mathbf{N a}+$ & Electric coupling & & Electric coupling & Hydration \\
\hline $\mathbf{C l}-$ & $\begin{array}{c}\text { Electric coupling } \\
\text { Cl- ads. to BSA }\end{array}$ & Electric coupling & & Hydration \\
\hline
\end{tabular}

\subsubsection{Hydration}

Hydration of solutes is incorporated by using a 'hydrated molar volume' that includes the volume occupied by water in the hydration layer of the solute molecule. For BSA, density measurements showed that its specific volume is $0.051 \mathrm{~m}^{3} / \mathrm{mol}$, which remains relatively constant within a $\mathrm{pH}$ range 
of $4.9-8$ [22]. Additionally, a single water monolayer $\left(0.028 \mathrm{~m}^{3} / \mathrm{mol} \mathrm{BSA}\right)$, corresponding to $0.4 \mathrm{~g}$ of water per $\mathrm{g}$ of BSA, is bound to the globular (spherical) protein, determining a total hydrated molar volume of $0.079 \mathrm{~m}^{3} / \mathrm{mol}[23,24]$. In the case of $\mathrm{Na}+$ and $\mathrm{Cl}-$, their hydrated molar volumes are calculated considering them to be spherical and using their corresponding Stokes radii, which already includes the water molecules that are bound to the ions.

This means that for the sake of simplicity in the calculations, the system is regarded as a mixture of hydrated (spherical) components and free water. $\bar{V}_{i}$ is the hydrated molar volume of the solutes and, consequently, the calculated $\varphi$ values include the volume of the water bound to the solutes.

\subsubsection{Electric coupling - Electroneutrality}

In general, the electro-neutrality of a multi-component solution containing species with charge Z, relative to a hydrogen ion, can be expressed as:

$$
\sum_{i}^{m-1} Z_{i} x_{i}=\sum_{i}^{m-1} Z_{i} \frac{\varphi_{i}}{\bar{V}_{i}}=0
$$

which should be included during the solution of the system of M-S Equations (Eqs. 9 and 11) to guarantee that in every position along the concentration polarization layer, the net charge remains zero.

\subsection{3 $\mathrm{Cl}^{-}$adsorption}

The adsorption of $\mathrm{Cl}$ - to BSA leads to an increase in the negative charge of BSA $\left(Z_{B S A}\right)$. In fact, $Z_{B S A}$ is the result of the difference between the bound protons $\left(v H^{+}\right)$and bound $\mathrm{Cl}^{-}\left(\mathrm{vCl}^{-}\right)$in the surface of the BSA molecule, which strongly depends on the $\mathrm{pH}$ and ion strength of the solution [25]:

$$
Z_{B S A}=v H^{+}-v C l^{-}
$$

in which $v \mathrm{H}^{+}$is calculated according to Tanford model for $\mathrm{H}^{+}$equilibria in BSA [26] and $v \mathrm{Cl}^{-}$is calculated following the two site chloride binding model of Scatchard et al. [25].

\subsubsection{Excluded volume}


2 Nevertheless, the aforementioned non-idealities are expressed in an implicit way within the model,

$$
\frac{\varphi_{i}}{R T} \nabla \mu_{i}=\varphi_{i} \frac{d \ln a_{i}}{d \varphi_{i}} \frac{d \varphi_{i}}{d z}
$$

6 The 'excluded volume' of a molecule is the volume that is inaccessible to other molecules due to the

finite size of the first molecule. When these molecules are forced close together at high concentrations, the osmotic pressure of the solution increases due to the resulting ordering of the molecules which

\section{Component 1: BSA}

To consider the thermodynamic effect of the excluded volume by the BSA molecules, the system can

$$
\begin{aligned}
& \frac{P V}{n_{1} R T}=\frac{1+\varphi_{1}+\varphi_{1}^{2}-\varphi_{1}^{3}}{\left(1-\varphi_{1}\right)^{3}} \\
& P=\Pi_{R S}=\frac{\varphi_{1} R T}{\bar{V}_{1}}\left(\frac{1+\varphi_{1}+\varphi_{1}^{2}-\varphi_{1}^{3}}{\left(1-\varphi_{1}\right)^{3}}\right)
\end{aligned}
$$

The activity of water is linked with the osmotic pressure in the following way.

$$
\ln a_{4}=\frac{-\bar{V}_{4} \Pi}{R T}
$$

Combining Eq. 16 and 17, we obtain:

$$
\ln a_{4}=-\frac{\bar{V}_{4}}{\bar{V}_{1}}\left(\frac{\varphi_{1}+\varphi_{1}^{2}+\varphi_{1}^{3}-\varphi_{1}^{4}}{\left(1-\varphi_{1}\right)^{3}}\right)
$$


1 The derivative of $\operatorname{lna}_{4}$ with respect to $\varphi_{1}$ can be obtained by applying the product and chain rules. The

2 result is shown in Eq. 19.

$$
\frac{d \ln a_{4}}{d \varphi_{1}}=-\frac{\overline{V_{4}}}{\bar{V}_{1}}\left(\frac{1+4 \varphi_{1}+4 \varphi_{1}^{2}-4 \varphi_{1}^{3}-\varphi_{1}^{4}}{\left(1-\varphi_{1}\right)^{4}}\right)
$$

3 To calculate the change in the activity of BSA from the change in water activity, the Gibbs-Duhem

4 relation can be used.

$$
S d T-V d P+\sum_{\substack{i=1 \\ i \neq 2,3}}^{m} n_{i} d \mu_{i}=0
$$

5 Considering that $\mathrm{T}$ and $\mathrm{P}$ are constants in the concentration polarization layer, the following relation

6 results for our 'imaginary' binary system:

$$
R T \sum_{\substack{i=1 \\ i \neq 2,3}}^{m} n_{i} d \ln a_{i}=0
$$

$7 \quad$ Which can be further simplified into:

$8 \quad n_{1} d \ln a_{1}=-n_{4} d \ln a_{4}$

$9 \quad \frac{\varphi_{1}}{\overline{V_{1}}} d \ln a_{1}=-\frac{\varphi_{4}}{\bar{V}_{4}} d \ln a_{4}$

$$
\varphi_{1} \frac{d \ln a_{1}}{d \varphi_{1}}=-\frac{\overline{V_{1}}}{\overline{V_{4}}}\left(1-\varphi_{1}\right) \frac{d \ln a_{4}}{d \varphi_{1}}
$$

Combining Eq. 22 with Eq. 19, we obtain:

$$
\varphi_{1} \frac{d \ln a_{1}}{d \varphi_{1}}=\frac{1+4 \varphi_{1}+4 \varphi_{1}^{2}-4 \varphi_{1}^{3}+\varphi_{1}^{4}}{\left(1-\varphi_{1}\right)^{3}}
$$

Eq. 23 represents the correction needed to account for the volume exclusion for component 1 (BSA).

12 By multiplying the right hand side of Eq. 23 by the gradient of molar volume $\left(\frac{d \varphi_{i}}{d z}\right)$, an expression equivalent to Eq. 14 is obtained, which should be used in the system of M-S Equations. 
1 At relatively high concentration of ions in the solution, the ions activity coefficient can be considered

2 constant since the observed changes in $\gamma_{2,3}$ can be explained by $\mathrm{Cl}^{-}$adsorption and BSA hydration;

3 only a small dependence on the protein concentration has been observed [29] [30, 31]. Therefore, Eq.

$4 \quad 14$ can be worked out in the following way for components 2 and 3 .

$$
\varphi_{i} \frac{d \ln a_{i}}{d \varphi_{i}}=\varphi_{i}\left(\frac{d \ln x_{i}}{d \varphi_{i}}+\frac{d \ln \gamma_{i}}{d \varphi_{i}}\right)
$$

5 Combining this with Eq. 5 we obtain:

$$
\varphi_{i}\left[\frac{d \ln \left(\frac{\varphi_{i}}{\bar{V}_{i} C_{T}}\right)}{d \varphi_{i}}+\frac{d \ln \gamma_{i}}{d \varphi_{i}}\right]=\varphi_{i}\left[\frac{\bar{V}_{i} C_{T}}{\varphi_{i}} \frac{d\left(\frac{\varphi_{i}}{\bar{V}_{i} C_{T}}\right)}{d \varphi_{i}}+\frac{d \ln \gamma_{i}}{d \varphi_{i}}\right]
$$

$$
\varphi_{i} \frac{d \ln a_{i}}{d \varphi_{i}} \frac{d \varphi_{i}}{d z}=\frac{d \varphi_{i}}{d z} \quad \text { for } i=2,3
$$$$
\text { for } i=2,3
$$

In reality, the activity coefficient of the ions are affected by the binding of water molecules to the solutes, and in the case of $\mathrm{Cl}^{-}$, its activity coefficient is also affected by the adsorption of $\mathrm{Cl}^{-}$to BSA. However, there is no need to account for these effects since they are already considered within the model: Water bound to the solutes is taken into account in their volume fraction and the adsorption of $\mathrm{Cl}^{-}$to BSA is already considered when calculating the charge of BSA and the electroneutrality along the concentration polarization layer [29]. As consequence, $\mathrm{Na}^{+}$and $\mathrm{Cl}^{-}$are considered thermodynamically ideal in the system and their activity coefficients are constant along the concentration polarization layer. 


$$
\begin{aligned}
& \mu_{i}^{w}=\mu_{i}^{\prime} \\
& R T \ln x_{i}^{w}+Z_{i} F \Psi^{w}=R T \ln x_{i}^{\prime}+Z_{i} F \Psi^{\prime} \quad \text { for } i=2,3
\end{aligned}
$$

8 By summing the expression corresponding for $i=2$ and $i=3$, Eq. 30 can be obtained, which is

$\ln x_{2}^{w}+\ln x_{3}^{w}=\ln x_{2}^{\prime}+\ln x_{3}^{\prime}$

$$
x_{2}^{w} x_{3}^{w}=x_{2}^{\prime} x_{3}^{\prime} \rightarrow x^{\prime}=\sqrt{x_{2}^{w} x_{3}^{w}}
$$

\subsection{Diffusion coefficients}

One of the advantages of the M-S approach is that the non-idealities are not contained in the $Đ$ coefficients but in the driving force for diffusion. Thus, the $Ð$ coefficients get less affected by changes in concentration than the Fick diffusion coefficients. In the case of BSA, $\bigoplus_{14}^{V}$ can be calculated using the following relation, which is derived in detail in the appendix section (A). The value for $D_{14}^{\infty}$ at diluted conditions is $6.1 \cdot 10^{-11} \mathrm{~m}^{2} / \mathrm{s}$. Notice that $\bigoplus_{14}^{V}$ is a function of only $\varphi_{1}$, without any influence from the electrical interactions between components.

$$
\bigoplus_{14}^{V}=D_{14}^{\infty} \frac{0.21+0.79 \exp \left(-4.7 \varphi_{1}\right)}{\frac{1+4 \varphi_{1}+4 \varphi_{1}^{2}-4 \varphi_{1}^{3}+\varphi_{1}^{4}}{\left(1-\varphi_{1}\right)^{3}}}
$$


1 Although the concentration of BSA can be very high in the concentration polarization layer $\left(\varphi_{1}^{w} \approx\right.$

2 0.55), the system is still diluted in terms of molar fractions, with $x_{4}^{w}$ values of nearly 0.99 . For this

3 reason, the effect of the cross diffusivities between solutes is not too important in the final outcome

4 [18]. The friction terms between BSA and the ions can be neglected in Eq. 9, while the cross

5 diffusivities between ions $\left(\bigoplus_{23}\right)$ can be calculated using the following empirical relation, in which $I$

6 stands for the ionic strength of the solution [18].

$$
\bigoplus_{23}=\bigoplus_{32}=\frac{\bigoplus_{24}+\bigoplus_{34}}{2} \frac{I^{0.55}}{\left|Z_{2} Z_{3}\right|^{2.3}}
$$

$7 \quad I=0.5 \sum_{i} Z_{i}^{2} x_{i}$

8 Note that the ion cross-diffusivity needs to be converted to modified diffusivities $\left(\bigoplus_{23}^{V}, \bigoplus_{32}^{V}\right)$ using the 9 relation in Eq. 8, before being used in the M-S Equation (Eq. 9). The binary diffusivities of $\mathrm{Na}^{+}$and $\mathrm{Cl}^{-}$with water can be assumed constant.

\subsection{Osmotic pressure}

When the flux in a specific point along the length of the membrane is lower than the local critical flux, steady state is still achieved but the chemical and electrical potential gradients in that point are not the maximal. Under these 'local subcritical conditions', change of phase for the proteins is not occurring because the gel concentration is not reached at the membrane surface. Therefore, the flux in that point is only influenced by the difference in the osmotic pressure across the membrane. Since it is assumed that no fouling occurs in the membrane surface at that specific point, the local membrane permeability is considered unaltered.

Under these conditions, the concentration of the protein at the membrane surface becomes an unknown because it cannot be considered that $\varphi_{1}^{w}=\varphi_{g e l}$. Thus, apart from the force balance represented by the M-S equations, an extra equation is needed. That extra equation is the flux relation derived from Darcy’s law (Eq.33). 


$$
J_{\text {osmo }}=L_{p}(\Delta P-\Delta \Pi)
$$

1

2 The osmotic pressure difference $(\Delta \Pi)$ in Eq.33 should be calculated using the real osmotic pressure of

$$
\begin{aligned}
& \Pi=-\frac{R T}{\varphi_{4}}\left(\frac{\ln \gamma_{4} \varphi_{4}}{\bar{V}_{4}}-\frac{\varphi_{1}}{\bar{V}_{1}}-\frac{\varphi_{2}}{\bar{V}_{2}}-\frac{\varphi_{3}}{\bar{V}_{3}}\right) \\
& \ln \gamma_{4}=\frac{\bar{V}_{4}}{\bar{V}_{1}} \frac{\varphi_{1}^{4}-3 \varphi_{1}^{2}}{\left(1-\varphi_{1}\right)^{3}}
\end{aligned}
$$

$$
\Pi=-R T\left(-2 C_{2,3}\right)
$$

Where $C$ stands for the concentration of the ions in $\mathrm{mol} / \mathrm{m}^{3}$. The factor 2 originates from the fact that the concentration of both ions is the same in the permeate, and is commonly known as Van't Hoff's index.

Figure 3 shows the predictions obtained using Eq. 34 and 35 for a solution of $\mathrm{BSA}, \mathrm{NaCl}$ and water at different $\mathrm{pH}$ and $\mathrm{I}=0.15 \mathrm{M}$. Experimental values from Vilker et al. are also shown in Figure 3, where it can be seen that the accuracy of the prediction is good [8]. At high volume fractions, the excluded volume of BSA increases exponentially and becomes the most important non-ideality in the system. Such behaviour is reflected in the steep rise in osmotic pressure as the system becomes more concentrated. 


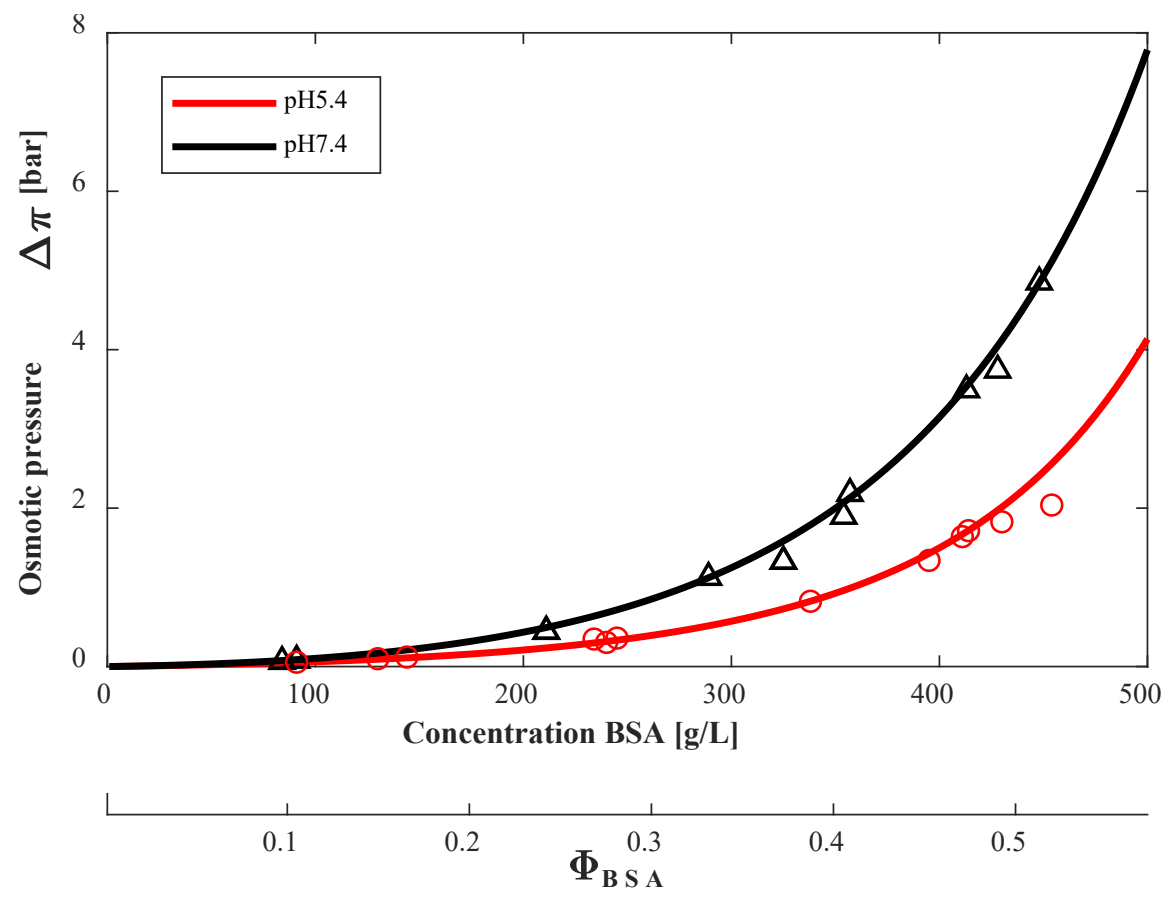

Figure 3. Osmotic pressure predictions (Eq.12, 13, 30, 34-36) and measurements for $\mathrm{BSA}+\mathrm{NaCl}+$ water solutions at $\mathrm{I}=0.15 \mathrm{M}$. The measurements were taken from the work of Vilker et al [8].

Higher osmotic pressures were calculated for higher $\mathrm{pH}$ values. This is considered in our model via the calculation of the charge of BSA, $Z_{B S A}$ (Eq.13), which becomes more negative as the $\mathrm{pH}$ increases. $Z_{B S A}$ influences the Donnan partition of the ions at the membrane interface, determining the presence of more counterions $\left(\mathrm{Na}^{+}\right)$at the retentate side to maintain the electro-neutrality in the system (Eq.12). This excess of ions determine an augmented osmotic pressure as compared with a solution at the same BSA concentration but at $\mathrm{pH}$ close to the isoelectric point.

\section{MATERIALS AND METHODS}

\subsection{Chemicals}

Milli-Q water ${ }^{\circledR}$ (ultrapure water) obtained from Millipak ${ }^{\circledR} 40$ Express Filter with a pore size of 0.22 $\mu \mathrm{m}$ (Darmstadt, Germany) was used for all the experiments. Lyophilized bovine serum albumin powder with a purity $\geq 96 \%$ was used to prepare the feed solutions. Likewise, $\mathrm{NaCl}$ with a purity $\geq$ 
$99 \%$ was used to set the ionic strength of the solutions. $\mathrm{NaOH}$ and $\mathrm{HCl}$ with a purity $\geq 99 \%$ were used to prepare solutions $2 \mathrm{M}$ to adjust the $\mathrm{pH}$ of the BSA solutions. All these chemicals were bought from Sigma Aldrich (Steinheim, Germany). Table 2 shows the charge, diffusivity at diluted conditions, radius and hydrated molar volume of the solutes used in this study.

Table 2. Properties of the solutes used in this study

\begin{tabular}{ccccc}
\hline Component & $\begin{array}{c}\boldsymbol{Z}_{\boldsymbol{i}} \\
{[]}\end{array}$ & $\begin{array}{c}\boldsymbol{D}_{\boldsymbol{i}}^{\infty} \\
{\left[10^{-10} \mathrm{~m}^{2} / \mathrm{s}\right]}\end{array}$ & $\begin{array}{c}\boldsymbol{r}_{\boldsymbol{i}} \\
{\left[10^{-9} \mathrm{~m}\right]}\end{array}$ & $\begin{array}{c}\overline{\boldsymbol{V}}_{\boldsymbol{i}} \\
{\left[10^{-5} \mathrm{~m}^{3} / \mathrm{mol}\right]}\end{array}$ \\
\hline $\mathrm{BSA}$ & $\begin{array}{c}\text { Dependent on } \\
\mathrm{pH} \text { and } I \\
\mathrm{Na}\end{array}$ & 0.61 & 3.14 & 7900 \\
$\mathrm{Cl}$ & -1 & 13.3 & 0.184 & 1.57 \\
& & 20.3 & 0.121 & 0.45 \\
\hline
\end{tabular}

\subsection{Membranes and set up}

Two types of UF flat sheet membranes were used during the experiments. Their pore size was such that complete rejection of $\mathrm{BSA}$ was assured while the rejection for $\mathrm{NaCl}$ was nearly 0 in the $\mathrm{pH}$ range $4.9-8$. The first membrane was a GH thin film membrane with a MWCO of 2500 Da produced by GE Water \& Process Technologies (Kent - United States), and the second one was a NP010 P polyether sulfone nanofiltration membrane with a $\mathrm{MWCO} \approx 3000$ Da produced by Microdyn Nadir (Wiesbaden, Germany). Experiments with pure water showed that the average membrane permeabilities $\left(L_{p}\right)$ of the membranes were $1.56 \times 10^{-6}$ and $2.79 \times 10^{-6} \mathrm{~m} /(\mathrm{s}$ bar $)$ for the GH and NP010P membrane respectively.

The rig consisted of a rectangular filtration channel with dimensions: 1000x50x7 mm, and included flow meters to measure crossflow and permeate flow, and sensors to monitor the temperature, electrical conductivity and pressure of the retentate and permeate streams. The pressure drop in the channel remained small ( $\sim 0.2$ bar) during experiments due to the large height of the channel.

A double jacket in the feed tank allows the stabilization of the system at $25^{\circ} \mathrm{C}$.

\subsection{Experiments at constant pressure}


$0.5 \% \mathrm{w} / \mathrm{w}$ feed solutions of BSA at different ion strength and $\mathrm{pH}$ were used in this study. To prepare the solutions, BSA and Milli-Q water were combined and stirred for 45 minutes at $25^{\circ} \mathrm{C}$. Then, the solutions were filtered with a Whatman ${ }^{\circledR}$ grade 50 filtration paper to remove aggregates and clumps. These feed solutions were adjusted to the desired ionic strength $(0.03$ and $0.15 \mathrm{M})$ using $\mathrm{NaCl}$. The experiments were run at three different $\mathrm{pH}$ values $(4.9,5.8$ and 7.2$)$, which were reached by adding aliquots of $2 \mathrm{M}$ solutions of $\mathrm{NaOH}$ or $\mathrm{HCl}$.

The filtration system was kept at $25{ }^{\circ} \mathrm{C}$ with a low crossflow flux of $0.056 \mathrm{~m} / \mathrm{s}$ to assure laminar flow inside the channel. The filtration started using a TMP of 2 bar, switching to 6 and 8 bar after reaching steady state flux. At steady state, samples from the retentate and permeate were collected to measure the concentration of BSA and $\mathrm{Cl}^{-}$. For each new experiment a new previously soaked membrane (GH or NP010) was used.

\subsection{Experiments at constant flux}

For the experiments at constant flux, the process parameters (temperature and crossflow velocity) were similar as in the constant-pressure experiments, but only the GH membrane was used. The pre-selected permeate flux was not altered during the experiment, and when the pressure reached a constant value, samples from retentate and permeate were collected. For each new experiment a new previously soaked membrane was used.

\subsection{Analytical methods}

High-performance liquid chromatography (HPLC) was used to measure the BSA concentration in the retentate and permeate streams. The analysis was done using a TSKGel G3000SWXL column (size: $300 \times 7.8 \mathrm{~mm}$ ) kept at a temperature of $30^{\circ} \mathrm{C}$. A solution of $30 \%$ Acetonitrile in MilliQ with $0.1 \%$ trifluoroacetic acid was used as eluent at a flow rate of $1 \mathrm{~mL} / \mathrm{s}$. UV detection at a wavelength of 214 nm was used to detect the protein. 
1 For the $\mathrm{NaCl}$ determination, $\mathrm{Cl}^{-}$was measured with Titralab AT1000 series from Hach (Tiel,

2 Netherlands), which is an automated titration equipment that uses Mohr's method to measure the concentration of $\mathrm{Cl}^{-}$ions by titrating them with $0.1 \mathrm{M} \mathrm{AgNO3.}$

\subsection{Computational Analysis}

MATLAB R2017b was used for all the calculations. Integrations were performed using the function 'trapz', which uses the trapezoidal numerical integration method. To solve the M-S equations, the function 'ode15i' was used, which allows solving systems of implicit differential equations.

\subsection{Algorithm}

Since no suitable model for the calculation of the local mass transfer coefficients for BSA was found in literature, the parameter $\delta(z=L)$ was fitted to experimental flux data at limiting conditions. From boundary layer theory, it is known that the boundary layer grows along the membrane axis with a proportionality of $(z / L)^{1 / 3}$. Therefore, with the value of $\delta_{(z=L)}$, the values of $\delta_{(z)}$ along the channel can be calculated, and consequently, the local critical fluxes along the membrane $J_{\text {crit }}(z)$ can also be obtained. The maximum protein concentration (gel concentration) was set to be $\varphi_{1}^{w}=0.55$ [12] and the observed rejection of ions was considered to be 0 regardless the process conditions.

$J_{c r i t}(z)$ are calculated by solving the M-S equations at every membrane position $z$, using the respective $\delta_{(z)}$ value. The system of M-S Equations described in Eq. 9 must be solved together with two extra conditions Eq. 11 and 12, and considering the bootstrap relation from Eq. 10. The term including the chemical potential differences in Eq. 9 should be worked out for each solute considering Eqs. 14, 23 and 27. Besides $J_{\text {crit }}(z)$, the concentration profiles for all 4 for components and the electrical potential $(\psi)$ along the concentration polarization layer can be estimated for every position $\mathrm{z}$.

The model is especially useful to estimate the mean permeate flux $\left(J_{v}\right)$ at 'sub-limiting' conditions, when $T M P<\Delta P_{\text {lim }}$ (region A or B in Figure 2). In such case, the next step is to calculate de critical distance $z_{\text {crit }}$, so we can distinguish two sections in the membrane: clean and fouled. To do so, we 
1 calculate $J_{\text {crit }}$ at the given TMP using Eq. 33, considering a concentration of $\varphi_{1}^{w}=0.55$, the original

2 permeability of the membrane and a distribution of ions according to Donnan equilibrium (Eq.30). The

3 obtained $J_{\text {crit }}$ is compared with the previously estimated $J_{\text {crit }}(z)$, and the point at which these two

4 fluxes are similar will be $z_{\text {crit }}$. If TMP is very small, it might be that the $J_{\text {crit }}$ value calculated with

5 Eq.33 is lower than all the $J_{\text {crit }}(z)$ along the membrane, then in that case the membrane is completely

6 clean (region A in Figure 2).

7 When the membrane is partially fouled (region B in Figure 2), from $z=z_{\text {crit }}$ to $z=L$, the membrane

8 is considered to be covered by gel; therefore, the local fluxes are the critical ones $\left(J_{\text {crit }}(z)\right)$, which

9 were previously calculated. On the other hand, from $z=0$ to $z=z_{\text {crit }}$, the membrane is not fouled, and the M-S Equations (Eq. 9, 11 and 12) should be solved once again to estimate the concentration of the solutes at the membrane surface. Since $\varphi_{1}^{w}$ is here an unknown, Eq. 33 is used as an extra equation to make possible the calculation of the local fluxes $J_{o s m o}(z)$. The $\delta_{(z)}$ values previously calculated at limiting conditions can be used since they can be considered constant regardless the pressure of the system. Finally, the mean permeate flux averaged over the entire membrane $\left(J_{v}\right)$ is calculated taking into account the $J_{\text {crit }}(z)$ and $J_{\text {osmo }}(z)$ values as described in Eq.3. For different conditions in the feed ( $\mathrm{pH}$ and ion strength), the complete algorithm should be repeated (see Figure 4). 
- Input: Experimental $J_{v, \text { lim }}$ at specific $\mathrm{pH}$ and I.

- Fit the model to find the value of $\delta(z=L)$.

- Calculate $\delta$ and $J_{\text {crit }}$ for all z values

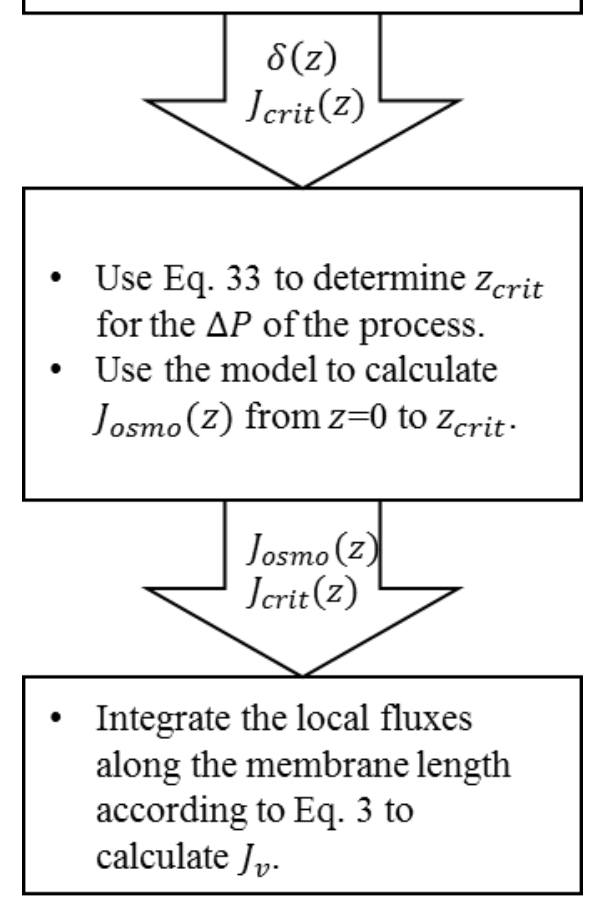

\section{RESULTS AND DISCUSSION}

\subsection{Filtration at limiting conditions}

Filtration experiments were performed at limiting conditions (region $\mathrm{C}$ in Figure 2) using feed solutions at different $\mathrm{pH}$ and ion strength. These variations imply different degrees of electrical interactions between solutes, which determine different permeate fluxes at steady state. The mass transfer coefficient $(k)$ and, consequently, the concentration polarization layer thickness were also expected to be different for every experiment. Therefore, the experimental steady state fluxes were used to estimate this thickness at the outlet of the system $\left(\delta_{(z=L)}\right)$. The results of this estimation are shown in Table 3. 
1 Table 3. Estimated values for $\delta_{(z=L)}$ from experimental flux data at limiting conditions for different $2 \mathrm{pH}$ and ion strength. GH membrane was used during these experiments.

\begin{tabular}{ccccc}
\hline \multicolumn{2}{c}{ Feed Solution } & $\boldsymbol{Z}_{\boldsymbol{B S A}}$ & $\boldsymbol{J}_{\boldsymbol{v} \text { lim }}\left[10^{-6} \mathrm{~m} / \mathrm{s}\right]$ & $\boldsymbol{\delta}_{(\mathbf{z}=\boldsymbol{L})}\left[10^{-4} \mathrm{~m}\right]$ \\
\hline $\mathrm{pH} 4.9$ & $I=0.03 \mathrm{M}$ & 0 & 2.24 & 1.34 \\
& $I=0.15 \mathrm{M}$ & -2.4 & 2.36 & 1.28 \\
$\mathrm{pH} 5.8$ & $I=0.03 \mathrm{M}$ & -6.7 & 2.90 & 1.30 \\
& $I=0.15 \mathrm{M}$ & -11.2 & 2.76 & 1.24 \\
$\mathrm{pH} 7.2$ & $I=0.03 \mathrm{M}$ & -13.5 & 3.48 & 1.68 \\
& $I=0.15 \mathrm{M}$ & -18.8 & 3.05 & 1.37 \\
\hline
\end{tabular}

According to film theory, $\delta$ is a function of the Fickian diffusivity $(\delta=D / k)$. Since D contains all the non-idealities of the system, it also changes with $\mathrm{pH}$ and $I$. Therefore, as we determined $\delta$ using experimental flux data, the effects of the non-idealities showed up in the value of $\delta$. Table 3 shows that a higher $\mathrm{pH}$ value leads, on average, to larger values of $\delta_{(\mathrm{z}=\mathrm{L})}$, while an increase in ionic strength at the same $\mathrm{pH}$ leads to lower $\delta_{(\mathrm{z}=\mathrm{L})}$ values.

As $Z_{B S A}$ increases due to a $\mathrm{pH}$ change, the repulsion between molecules increases as well, reducing the thickness and the density of the gel layer, and, as consequence, determining a higher $J_{v} \lim$. [34]. Ion strength plays also an important role in this matter, since the increase of ions in the system from $0.03 \mathrm{M}$ to $0.15 \mathrm{M}$ screens the electrostatic interactions between BSA molecules, reducing the repulsion.

Although only one value for $\delta$ was fitted $\left(\delta_{(z=L)}\right)$, the calculation of local critical fluxes along the entire membrane $J_{\text {crit }}(\mathrm{z})$ was necessary . The obtained results for the different physicochemical conditions are plotted in Figure 5. 


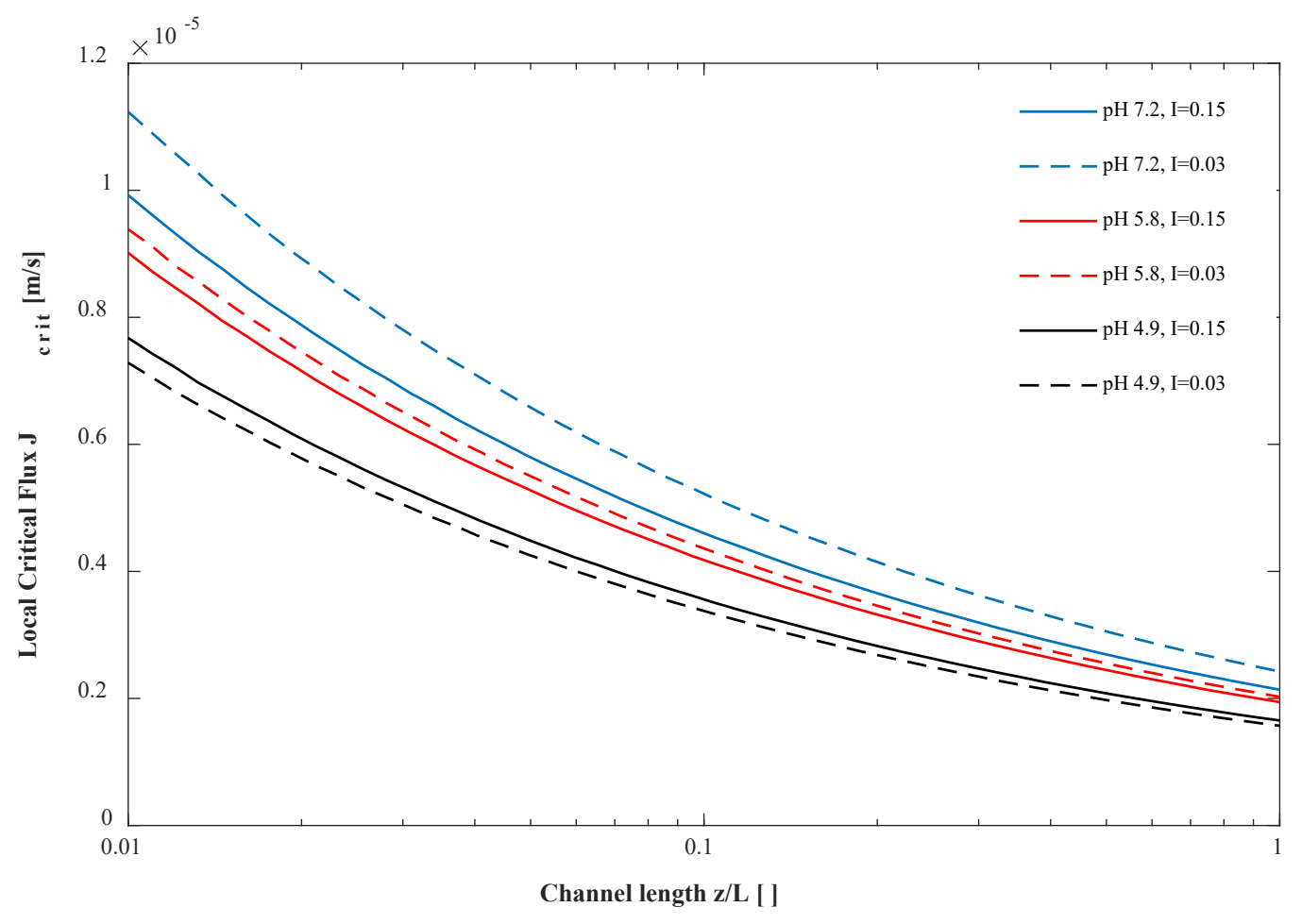

Figure 5. Local Critical fluxes $J_{\text {crit }}(z)$ along the channel length for different $\mathrm{pH}$ and ion strength values. In all the cases the feed was composed by BSA $(0.5 \mathrm{~g} / \mathrm{L}), \mathrm{NaCl}$ and water.

The local critical fluxes varied strongly along the membrane regardless the feed used in the experiment. This was due to the expression used to represent the thickening of the boundary layer along the membrane (Eq.1).

As expected, higher local critical fluxes were obtained at higher $\mathrm{pH}$ values (higher negative charge for $\left.Z_{B S A}\right)$. At pH 5.75 and 7.25, increasing ion strength leads to lower fluxes due to the screening of the electrical repulsion between BSA molecules, usually represented as thinner electrical double layers around the charged macromolecules. At the isoelectric point (pH4.9), the opposite behaviour was observed: The local fluxes were slightly higher at higher ion strength. At this $\mathrm{pH}$, the number of positive and negative charges at the surface of BSA are the same, and their spatial distribution over the protein surface leads to intermolecular attraction and a compact gel. The increment of ions in the solution screened this attraction, leading to a higher permeate flux [33]. 
1 The way how BSA and ions influence each other can be seen in Figure 6, in which the concentration

2 profiles of the solutes are shown for a specific position in the filtration channel $(z / L=0.33)$. The BSA

3 concentration grows exponentially in the concentration polarization layer towards the membrane

4 surface, until it reaches its maximum at $\delta=113 \mu \mathrm{m}$, and form a gel. The steep increase of BSA

5 influences the local concentration of the other ions, since electroneutrality must be maintained along

6 the whole system. As a result, the concentration of the counterion $\mathrm{Na}^{+}$increases to compensate the negative charged BSA, while the concentration of the co-ion $\mathrm{Cl}^{-}$decreases. Given that the BSA concentration is assumed constant in the gel layer, the concentration of ions was also assumed constant over the gel layer thickness. Additionally, local thermodynamic equilibrium was assumed at the membrane surface (Eq. 28-30) to calculate the ions concentration just inside the membrane. These concentrations are similar to the concentrations in the permeate stream since the membrane pores were considerably bigger than the ions, so the friction of the transient ions with the membrane walls was considered negligible.

To enable the solution of the M-S equations, free passage of the ions through the membrane $\left(R_{N a C l}=0\right)$ and total BSA rejection was assumed $\left(R_{B S A}=1\right)$ for the calculations of the solute fluxes (Eq.10). These assumptions were experimentally checked at all feed conditions used in this study. As shown in Figure 6, the calculated $\mathrm{NaCl}$ rejection coincides with our initial assumption, it fluctuated between 0.05 and 0.05 , while $R_{B S A}$ was never lower than 0.99 . 


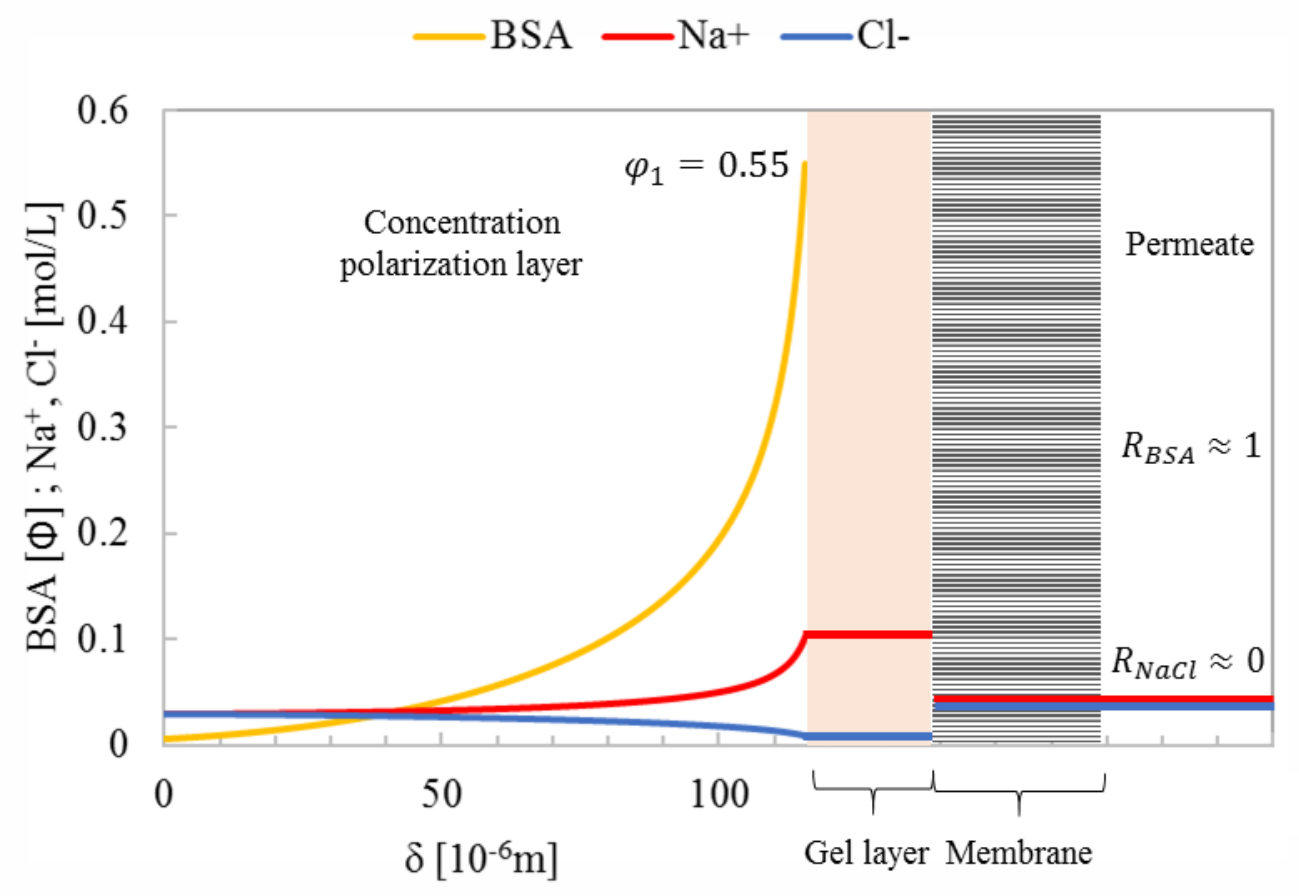

Figure 6. Solutes' concentration profiles corresponding to the position $z / L=0.33$ in an ultrafiltration channel at limiting conditions. The feed solution contained $0.5 \% \mathrm{w} / \mathrm{w}$ BSA and was at $\mathrm{pH} 7.2$ and $I=0.03 \mathrm{M}(\mathrm{NaCl})$. The thickness of the gel layer and the membrane are not plotted to scale with respect to the thickness of concentration polarization layer.

The gel layer constitutes an extra resistance for the flow of liquid through the membrane. This is, however, not relevant for our model, since the local critical fluxes are determined by the force equilibrium in the concentration polarization layer.

\subsection{Filtration in the Transition region}

When $T M P<\Delta P_{\text {lim }}$, some area of the membrane remains clean. As there is no gel layer formation on this area, the local membrane permeability $\left(L_{p}\right)$ is considered constant. Thus, the local permeate flux here is only influenced by the effect of the osmotic pressure. Since the solutes concentrations at the surface of the uncovered section of the membrane are different depending on the position along the membrane, the osmotic pressure is expected also to vary along the membrane length. For this reason, the M-S Equations had to be solved at every position $z$, considering $\delta_{(z)}$ to be the same as the values determined under limiting conditions. Figure 7 shows the estimated local fluxes obtained when a membrane was partially fouled. As consequence, two mechanisms (osmotic pressure and gel layer) coexisted within a filtration channel. In physical terms, the only difference between them is the 


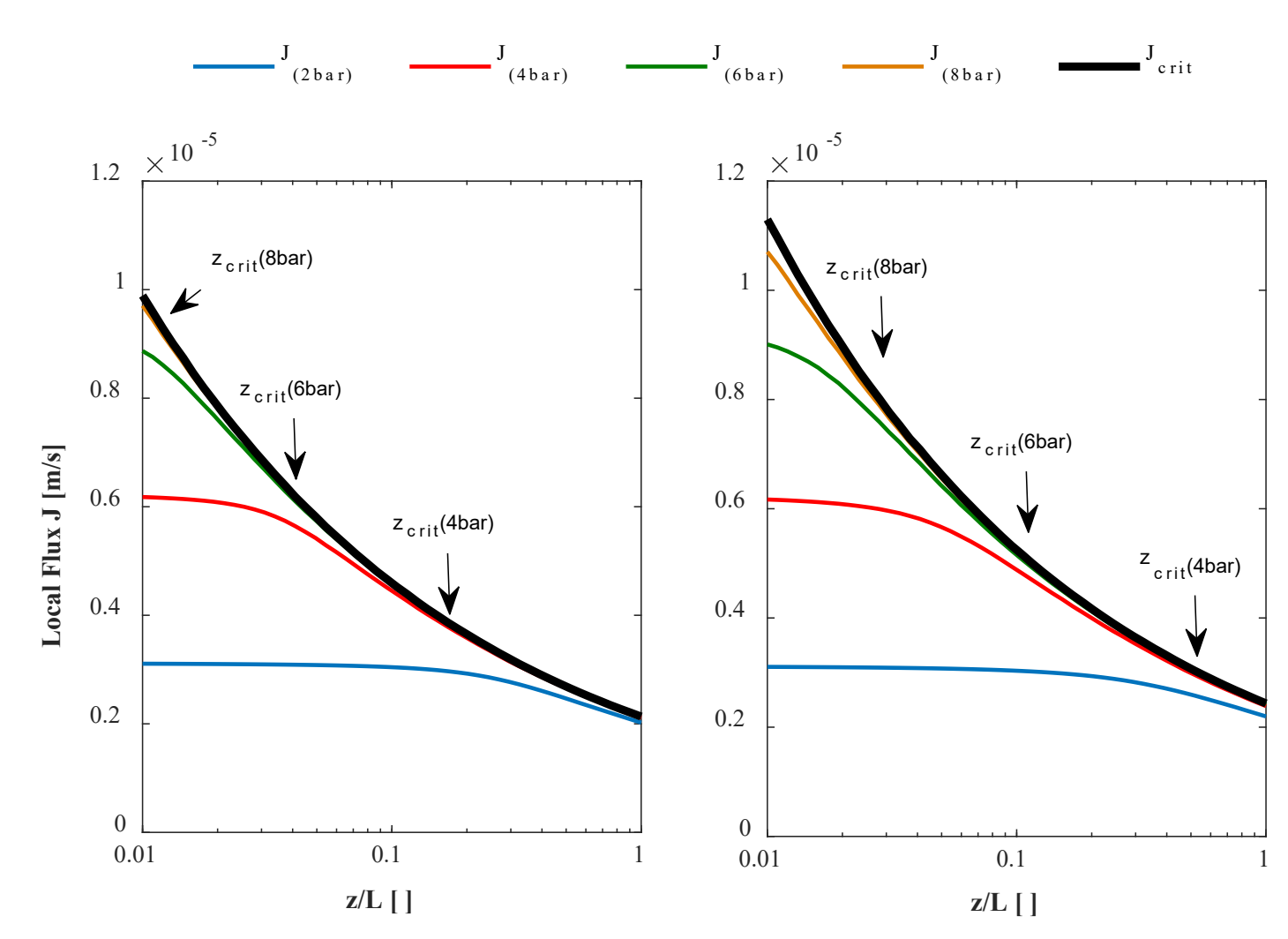

formation of the gel layer. In this study, however, the strategy to calculate $J_{v}$ differs depending on the mechanism involved. The critical distance $\left(z_{c r i t}\right)$, at which fouling starts at the membrane, separates both mechanisms and was different depending on TMP.

Figure 7. Local permeate fluxes due to two mechanisms (osmotic pressure and gel layer) during the desalination of a $0.5 \% \mathrm{w} / \mathrm{w}$ BSA solution using a GH membrane at $\mathrm{pH} 7.2$ and ion strength of $I=0.15$ $\mathrm{M}$ (left) and $I=0.03 \mathrm{M}$ (right). Fluxes from $z=0$ to $z_{\text {crit }}$ are calculated considering the local osmotic pressures of the system (Eq.33). Fluxes from $z_{\text {crit }}$ to $z=L$ are similar to $J_{\text {crit }}$, as obtained in section 4.1. $\mathrm{X}$ axis was plotted in logarithmic scale to show clearer the transition between mechanisms.

As the pressure increases, the resulting local fluxes increase especially at the entrance of the channel, at which the maximum local flux (the critical flux) is higher. For the estimations at $I=0.15 \mathrm{M}$ (Figure 7 - left), gel formation is just about to occur at the outlet of the membrane at a pressure of 2 bar. At a pressure of 8 bar, the membrane is almost completely fouled and the limiting flux is nearly reached. Therefore, pressures higher than 8 bar do not alter the steady state permeate flux of the system. At lower ionic strength (Figure 7 - right), higher values for the critical fluxes and, consequently, for $z_{\text {crit }}$ 
were obtained. Gel formation appears at a higher pressure than with the higher ionic strength of $I=0.15 \mathrm{M}$, and the limiting flux is not yet achieved at a pressure of $8 \mathrm{bar}$. These results agree with the DLVO theory with respect to the screening effect of ions, and go in line with what has been observed experimentally by many authors for decades $[4,34]$.

It has been measured by other authors that for a given BSA concentration, the osmotic pressure gets lower as the ionic strength increases [35, 36]. This would produce higher fluxes at higher $I$.

Interestingly, this effect was not visible in the 'uncovered' membrane sections shown in Figure 7, in which $J_{\text {osmo }}(z)$ was always lower for $I=0.15 \mathrm{M}$ than for $I=0.03 \mathrm{M}$. The reason is that both figures (Figure 7 left and right) are not comparable for this matter. BSA concentrations at the membrane in the uncovered regions are much lower for $I=0.03 \mathrm{M}$. The reason is that, at low ionic strength, the electrical potential gradient is greater (see Figure 8). This promotes the back diffusion towards the bulk of the retentate, lowering the increase of the BSA concentration.
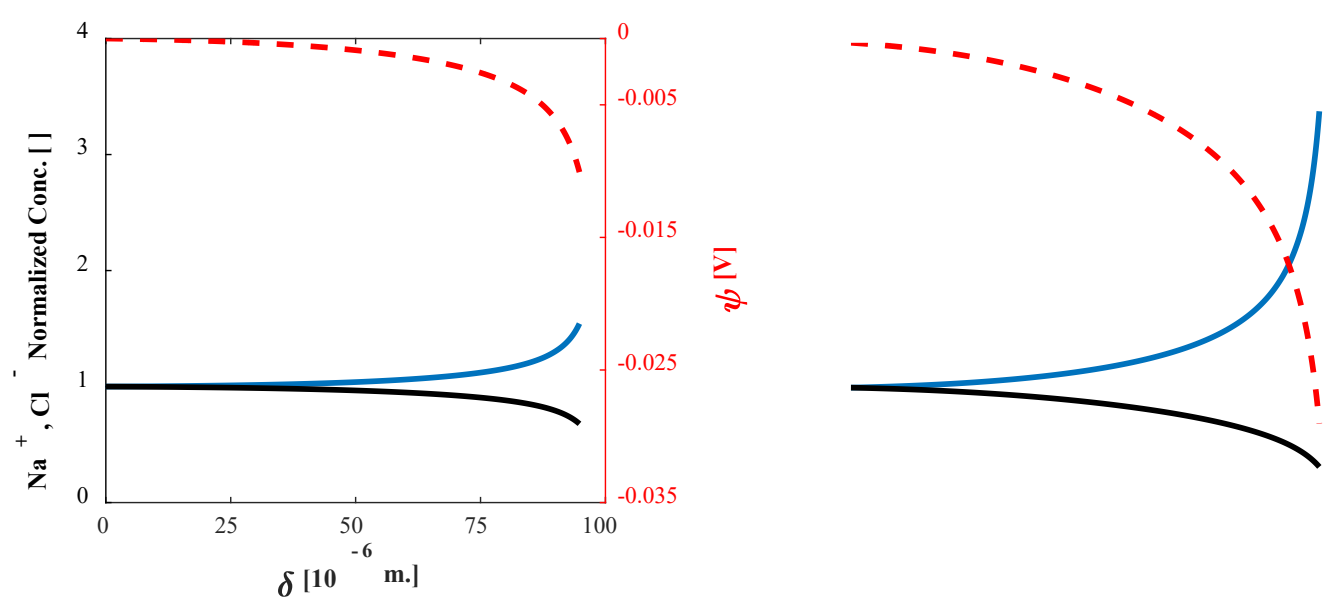

Figure 8. Normalized concentration profiles for $\mathrm{Na}^{+}$and $\mathrm{Cl}^{-}$along the concentration polarization layer during the UF of $0.5 \% \mathrm{w} / \mathrm{w}$ BSA. The results correspond to the position $z / L=0.33$. The $\mathrm{pH}$ of the solutions was 7.2 and ion strength was $I=0.15$ (left) and $I=0.03$ (right). The resulting electrical potential is represented with dashed lines.

Figure 8 shows a comparison in the behaviour of the accompanying ions at two different ionic strengths. As the concentration of BSA increases along the concentration polarization layer, the concentration of $\mathrm{Na}^{+}$increases and that of $\mathrm{Cl}$ - decreases. This change in concentrations is much 
stronger at low ionic strengths. Therefore, this excess of $\mathrm{Na}^{+}$ions explains why higher osmotic pressures are observed under these conditions, compared to those at high ionic strengths. Another consequence of this asymmetric distribution of ions is that the electrical potential is much more negative at low ionic strengths, determining, as explained in Figure 6, an enhanced back diffusion of BSA towards the retentate bulk. Consequently, by considering the electroneutrality condition (Eq.12) during the resolution of the M-S Equations and imposing a Donnan partitioning across the membrane (Eq.30), we can accurately calculate the osmotic pressure in any position along the system for any $\mathrm{pH}$ and $I$. The resulting quantification of the electrical potential is an advantage of using the M-S Equations over other models where the electrical interactions between components are represented as an additional factor.
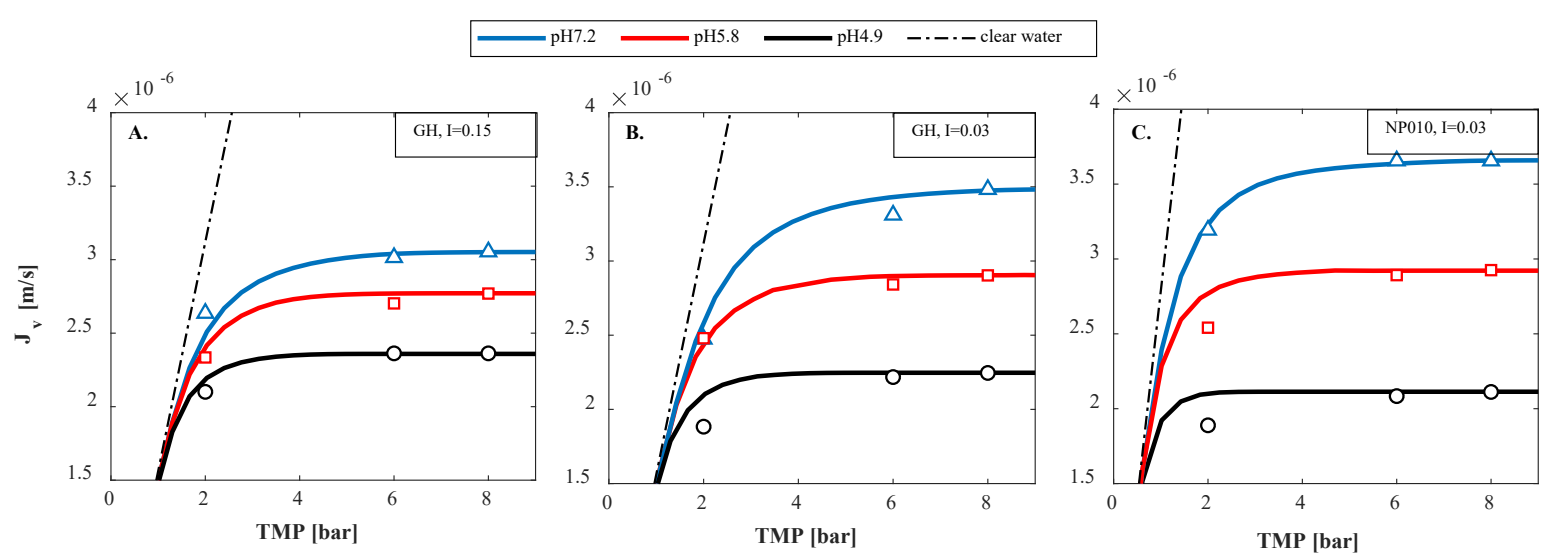

Figure 9. Permeate flux for a solution of $0.5 \% \mathrm{w} / \mathrm{w}$ BSA at different $\mathrm{pH}$ and $I$ for two different membranes (GH and NP010). Continuous lines represented the model estimations and markers are the experimental measurements at steady state. Dash-dotted lines are the fluxes using clear water.

The model was experimentally verified with measurements of the overall permeate flux under different physicochemical conditions (Figure 9). In general, a good accuracy was obtained for all the experiments. As expected, higher fluxes were obtained at lower ionic strengths, with the exception of the measurements at the isoelectric point, where the excess of ions screens the attraction between BSA molecules, resulting in a higher flux at high $I$. 
1 When comparing the effect of different membranes (Figure $9 \mathrm{~B}$ and C), somewhat similar limiting

2 fluxes were obtained, regardless of the large difference in water permeability between the GH and the

3 NP010 membranes. This similarity was expected as the local critical fluxes are determined by the equilibrium of forces in the concentration polarization layer, and not by the membrane. The same type of experimental observations have been reported by other authors $[4,37]$. When working with fluxes lower than the limiting flux (Transition region), some parts of the membrane remain uncovered and, consequently, some differences appeared in the flux due to the different permeability between both membranes. The GH membrane required higher pressure to reach the limiting flux, especially at $\mathrm{pH}$ 4.9 and 5.8 .

\subsubsection{Comparison with a simplified approach}

In order to compare the predictions of our model with a more traditional approach, extra calculations were made using a simplified model considering a constant Fick diffusion coefficient. In this simplified interpretation, only the effects of osmotic pressure are considered and the presence of the gel layer is neglected. Likewise, the thickness of the boundary layer was considered to be uniform, and average values for $\delta$ were estimated from the $\delta_{(z)}$ values obtained in section 4.1. As a result, $J_{v}$ was calculated from the following system of equations, in which the concentration of BSA at the membrane $\left(C_{w}\right)$ is also unknown.

$$
\begin{aligned}
& J_{v}=\frac{D}{\delta} \ln \frac{C_{w}}{C_{b}} \\
& J_{v}=L_{p}(\Delta P-\Delta \Pi)
\end{aligned}
$$

Eq. 37 represent the traditional film model, and Eq. 33 the flux equation based on Darcy's law, which was already introduced in section 2.6. Here, the original values of $L_{p}$ were used and $\Delta \Pi$ was calculated according to Eqs. 34-36. Fick diffusivities of $3.9 \cdot 10^{-11}$ and $7.8 \cdot 10^{-11} \mathrm{~m}^{2} / \mathrm{s}$ were obtained from literature for $\mathrm{pH} 4.9$ and 7.2 respectively $[38,39]$. These values correspond to experimental measurements at $I=0.15 \mathrm{M}$ and molar fraction $\varphi_{1}=0.2$, which was considered to be an average value in the polarization layer. 
1 Figure 10 shows the estimations for the permeate flux $J_{v}$ and molar fraction at the membrane surface

$2 \varphi_{w}$. In line with the experimental data, higher $J_{v}$ values were obtained at $\mathrm{pH} 7.2$ than at $\mathrm{pH} 4.9$ due to

3 the higher Fick diffusivity at higher $\mathrm{pH}$. This simplified model, however, is not able to represent the

4 effect of ionic strength in the system, since $D$ was considered constant at any $I$ value. At $\mathrm{pH} 7.2, J_{v}$ is

5 calculated to be higher at $I=0.15$ than at $I=0.03$, when the opposite has been experimentally

6 demonstrated. Therefore, for this simplified model to make sense, it would be necessary to use specific

$7 \quad D$ values measured under every single condition tested in the system. The advantage of the MS

8 approach is that the non-idealities contained in $D$ can be calculated while solving the concentration

9 profiles in the concentration polarization layer. As shown in Figure 8, the resulting electrical potential

10 influences the driving force and no experimental values for $D$ are needed. These non-idealities are not

11 constant but depend on the position in the concentration polarization layer since they are affected by

12 protein and ion concentrations. These aspects are clearly not considered in this simplified representation and the accuracy of the predictions is, therefore, affected. 

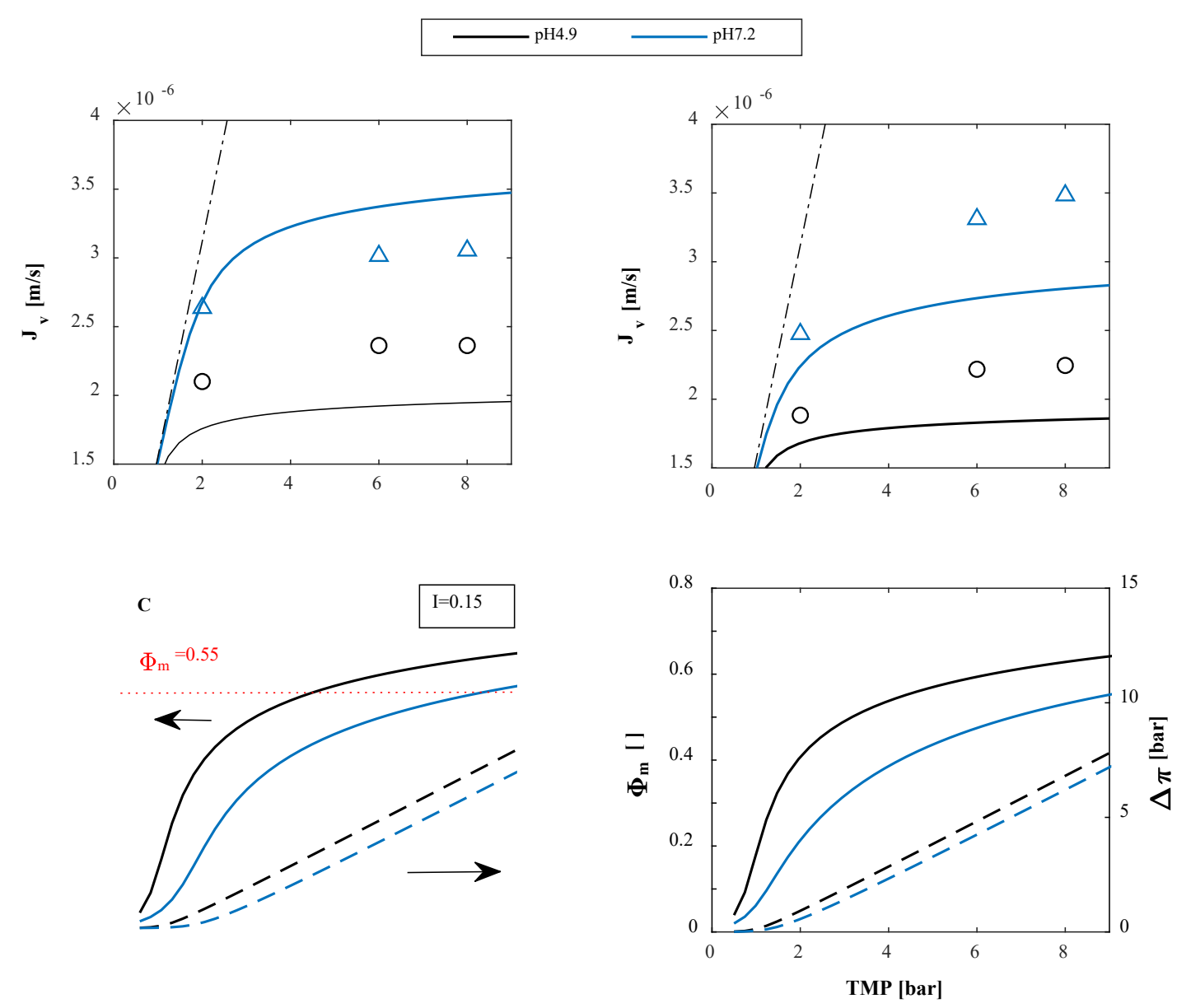

Figure 10. Permeate flux estimations for the UF of BSA (Figure A and B) using a simplified model based on constant Fick diffusivities $\left(D_{p H 4.9}=3.9 \cdot 10^{-11}\right.$ and $D_{p H 7.2}=7.8 \cdot 10^{-11} \mathrm{~m}^{2} / \mathrm{s}$. $)$. The resulting BSA volume fractions at the membrane $\varphi_{w}$ (continuous lines) and the osmotic pressure difference over the membrane $\Delta \pi$ (dashed lines) are represented in Figure $\mathrm{C}$ and $\mathrm{D}$. The system conditions were similar as in Figure 9, except that here only the results for GH membrane are shown.

This simplified model only considers the presence of BSA, while the effect of having other solutes in the mixture is entirely contained in the $D$ value. On the other hand, the MS approach presented in this paper considers every species in the system and can be expanded easily to more components without the need of new $D$ values for BSA specific for the new conditions. Additionally, the MS approach opens the possibility of calculating the rejection of these extra solutes that most of the times are part of the buffer solution accompanying the proteins.

Another drawback of this simplified approach is that, at high pressures, the values obtained for $\varphi_{w}$ are higher than the gel volume fraction $\left(\varphi_{g e l}=0.55\right)$ (Figure $10-\mathrm{C}$ and D). Such unreal concentrations 
are calculated by the model to produce higher $\Delta \pi$ values to compensate the fact that the presence of the gel layer is neglected. As expected, the gel concentration is reached at lower pressures at $\mathrm{pH} 4.9$ due to the lower repulsion between BSA molecules.

In general, it can be stated that besides being inaccurate due to the constant $\mathrm{D}$, the simplified model is only suitable at very low permeate fluxes when no gel layer is formed; this is demonstrated by the fact that the $J_{v}$ predictions are more accurate at $\mathrm{TMP}=2$ bar than at higher pressures for both $\mathrm{pH}$ values.

\subsection{Experiments at constant flux}

In industry, membrane filtration is mostly done at constant flux. Experiments under these conditions were performed to evaluate the applicability of our model. Figure 11 shows experimental measurements of TMP as function of time for different feed conditions. The left hand graph shows the difference in the pressure behaviour over time when the permeate flux was set higher and lower than the limiting flux. In the former case, no steady state was achieved and the pressure kept growing quickly, while in the latter case the pressure increased gently until it reached a plateau after few hours. This plateau represents the steady state pressure, which is the equilibrium point where the drag due to convection is equal to the back diffusion effects due to the chemical and electrical potentials in the concentration polarization layer.
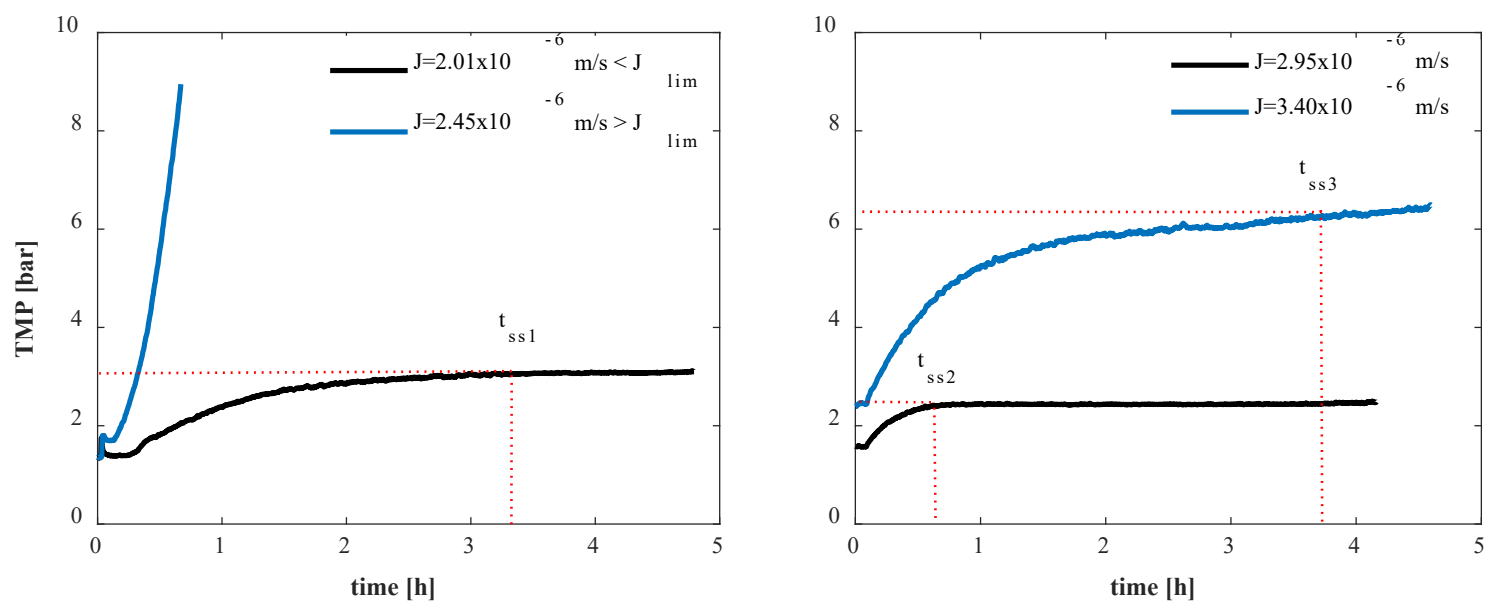

Figure 11. TMP as function of time for experiments at constant flux using GH membrane. The physicochemical conditions of the $0.5 \% \mathrm{w} / \mathrm{w}$ BSA solution were $\mathrm{pH}$ at $4.9, I=0.03$ (left), and $\mathrm{pH}=7.2$, $\mathrm{I}=0.03$ (right). 
In the right-hand side of Figure 11, the comparison of the TMP evolution over time for two different permeate flows is shown. At a permeate flux of $2.95 \times 10^{-6} \mathrm{~m} / \mathrm{s}\left(15 \%\right.$ lower than $\left.J_{\text {lim }}\right)$, steady state was achieved in less than one hour, while at a higher flux ( $3 \%$ lower than $\left.J_{\text {lim }}\right)$ it took almost 4 hours to reach it. This depicts the growth of the gel layer along the filtration channel over time. It can be interpreted as fouling occurring only in the outlet of the channel at low fluxes, and growing towards the channel inlet as the flux increases. Additionally, the fact that the increase of TMP becomes smaller over time shows that the system was stable and will eventually reach a steady state.

With the experimental measurements presented in Figure 11 is evident that below the limiting flux, in the transition region, fouling is already taking place over the membrane, as properly represented in our model. Consequently, it is incorrect to simplify the description of UF to a system that can go from 'completely clean' to 'completely fouled' in one instant. The transition region is quite important, and very relevant for practical UF operation.

At steady state, the combination of permeate flux and TMP are the same whether we operate the system at constant pressure or constant flux. Therefore, our model can be easily adapted to predict the steady state TMP, using as input the required value for the permeate flux. Figure 12 shows the accuracy of these estimations.

It is important to mention, however, that when the permeate flux was set close to $J_{\text {lim }}$, it was somewhat difficult to experimentally identify a steady state value for the TMP. The gel layer kept growing slowly and sometimes irregularly, implying that the duration of one experimental run might not capture the complete process of gel layer growth. Therefore, the next step would be to investigate the kinetics of the formation and growth of the gel layer. This will then enable the reliable prediction of the system performance over very long production runs. 


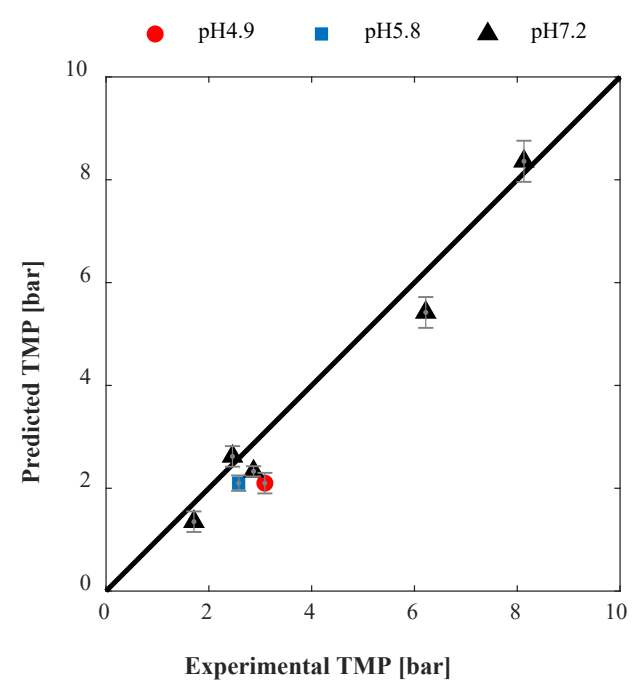

Figure 12. Comparison between model estimations of TMP and TMP experimental measurements for the UF of BSA performed at constant flux and $I=0.03 \mathrm{M}$.

In this study we considered the 'uncovered section' of the membrane to be completely clean; hence, its original permeability was used in the calculations. This is obviously an over-simplification. Over time BSA will adsorb to the membrane due to the intermolecular interactions with the membrane surface, reducing slightly the local permeability. Since our model could well estimate the experimental results, this effect was negligible under the conditions that were chosen and our assumption was justified; however, this may be different with other conditions or during longer filtration runs. 
1

2

3

4

5

6

\section{CONCLUSIONS}

A model to estimate the permeate flux at steady state of a UF system was developed taking into account not only the concentration profiles of the protein (BSA) but also the concentrations of the accompanying ions. The model was developed using modified Maxwell Stefan Equations expressed as function of the components' volume fraction, which allowed the easier consideration of the nonidealities of the system (hydration, adsorption, electrical interactions and volume exclusion).

The advantage of the proposed model is that the non-idealities of the system can be rigorously calculated by considering the local concentration of the components. In that way, no extra data sets on Fick diffusivities are needed and the approach is suitable for a large range of system conditions.

It was found that more than one mechanism can influence the permeate flux in a filtration system. For partially fouled membranes, two sections of the membrane can be distinguished: The uncovered section is influenced only by the local osmotic pressure difference, while in the section covered with gel, local critical fluxes, defined by the gel layer mechanism, are attained.

The Maxwell-Stefan approach has thus proved effective in calculating the concentration profiles of the charged solutes along the concentration polarization layer. Therefore, it opens the future possibility to calculate not only the rejection of the proteins, but also that of the ions, which are frequently present in the background solutions, considering the coupled mechanisms that influence the transport of the charged molecules. 
2 This work was carried out as part of a project of the Institute for Sustainable Process Technology

3 (ISPT), The Netherlands: project number CM-20-05. We thank Bobby Oka Mahendra and Desiree

4 Nieuwenhuijse for their contribution in the experimental part of this study within their Master thesis

5 project at the Food Process Engineering group (Wageningen University).

\section{$7 \quad$ Nomenclature}

$8 \quad a$

Chemical activity [ ]

$9 \quad C_{g}$

Gel concentration $\left[\mathrm{mol} / \mathrm{m}^{3}\right]$

10

$C_{m}$

Concentration at the membrane $\left[\mathrm{mol} / \mathrm{m}^{3}\right]$

$11 C_{T}$

Total molar concentration $\left[\mathrm{mol} / \mathrm{m}^{3}\right]$

$12 D$

Fick Diffusion coefficient $\left[\mathrm{m}^{2} / \mathrm{s}\right]$

$13 \quad F$

Faraday constant [Coulomb/mol]

$14 I$

Ion strength in Eq. 32[ ]

15

Volumetric Flux $[\mathrm{m} / \mathrm{s}]$

Local critical flux [m/s]

Local flux determined only by the osmotic pressure $[\mathrm{m} / \mathrm{s}]$

Mean flux averaged over the membrane $[\mathrm{m} / \mathrm{s}]$

Mass transfer coefficient $[\mathrm{m} / \mathrm{s}]$

Total length of the filtration channel [m]

Membrane permeability [m]

number of moles [mol]

Total number of components in mixture (including water as component $m$ ) [ ]

Pressure [bar]

Permeate

Gas constant $[\mathrm{J} /(\mathrm{K} \mathrm{mol})]$ 


$\begin{array}{llll}1 & r & \text { radius }[\mathrm{m}] \\ 2 & \mathrm{~S} & \text { Entropy }[\mathrm{J} / \mathrm{K}] \\ 3 & T & \text { Temperature }[\mathrm{K}] \\ 4 & T M P & \text { Transmembrane Pressure }[\mathrm{Pa}] \\ 5 & u & \text { Linear velocities }[\mathrm{m} / \mathrm{s}] \\ 6 & V & \text { Volume }\left[\mathrm{m}^{3}\right] \\ 7 & \bar{V} & \text { Molar volume (hydrated) }\left[\mathrm{m}^{3} / \mathrm{mol}\right] \\ 8 & x & \text { Molar fraction [ ] } \\ 9 & w & \text { Membrane surface } \\ 10 & Z & \text { Charge [ ] } \\ 11 & Z & \text { Position along the length of the filtration channel }[\mathrm{m}] \\ 12 & z_{\text {crit }} & \text { Critical distance [ ] }\end{array}$

\section{Greek letters}

Activity coefficient [ ]

$17 \quad \Pi$

Osmotic Pressure [Pa]

$18 \Pi_{R S}$

Osmotic Pressure due to excluded volume (Rigid Sphere)[Pa]

$19 \delta$

Concentration polarization layer thickness [m]

20

Maxwell-Stefan diffusion coefficient $\left[\mathrm{m}^{2} / \mathrm{s}\right]$

$21 \bigoplus^{V}$

Modified Maxwell-Stefan diffusion coefficient $\left[\mathrm{m}^{2} / \mathrm{s}\right]$

$22 \Gamma$

Thermodynamic factor [ ]

Chemical Potential [J/mol]

$24 \tilde{\mu}$

Electrochemical Potential [J/mol]

$25 \varphi$

Volume fraction [ ]

$26 \psi$

Electrical potential [V] 


\section{APPENDICES}

2 A. Calculation of the modified M-S diffusion coefficient $\bigoplus_{14}^{V}$

3 The M-S diffusion coefficient of BSA(Đ) is related to the Fick diffusion $(D)$ coefficient in the

4 following way:

$$
D_{i j}=\bigoplus_{i j} \Gamma_{i}
$$

5 In which $\Gamma$ is the thermodynamic factor that corrects for the non-idealities of the system. In the case of

6 BSA, $\Gamma$ can be expressed as follows to account for volume exclusion:

$$
\Gamma_{11}=x_{1} \frac{d \ln a_{1}}{d x_{1}} \frac{d \varphi_{1}}{d \varphi_{1}}=x_{1} \frac{d \ln a_{1}}{d \varphi_{1}} \frac{d \varphi_{1}}{d x_{1}}
$$

7 Considering the following approximations:

$$
\begin{aligned}
& C_{T} \approx \frac{\varphi_{4}}{\bar{V}_{4}} \\
& \varphi_{4} \approx\left(1-\varphi_{1}\right)
\end{aligned}
$$

$8 \quad$ A new expression for $x_{i}$ can be obtained:

$$
x_{i}=\frac{\varphi_{i}}{\bar{V}_{i} C_{T}} \approx \frac{\varphi_{i} \bar{V}_{4}}{\bar{V}_{1}\left(1-\varphi_{1}\right)}
$$

9 Eq. A5 for the case of BSA can be differentiated with respect to $\varphi_{1}$ using the quotient and chain rules:

$$
\frac{d x_{1}}{d \varphi_{1}}=\frac{\bar{V}_{4}}{\bar{V}_{1}} \frac{1}{\left(1-\varphi_{1}\right)^{2}}
$$

10

Combining Eq. A2 A5 and A6:

$$
\Gamma_{11}=\frac{\varphi_{i} \bar{V}_{4}}{\bar{V}_{1}\left(1-\varphi_{1}\right)} \frac{d \ln a_{1}}{d \varphi_{1}} \frac{\bar{V}_{1}\left(1-\varphi_{1}\right)^{2}}{\bar{V}_{4}}=\left(1-\varphi_{1}\right) \varphi_{1} \frac{d \ln a_{1}}{d \varphi_{1}}
$$

11

Plugging Eq. A7 into Eq. A1:

$$
\bigoplus_{14}=\frac{D_{14}}{\left(1-\varphi_{1}\right) \varphi_{1} \frac{d \ln a_{1}}{d \varphi_{1}}}
$$


1 Considering Eq. 8, A3 and A4, the expression for the modified M-S diffusivity $\bigoplus_{14}^{\mathrm{V}}$ can be obtained, as

2 shown in Eq. A9.

$$
\bigoplus_{14}^{V}=\bigoplus_{14}\left(1-\varphi_{1}\right)=\frac{D_{14}}{\varphi_{1} \frac{d \ln a_{1}}{d \varphi_{1}}}
$$

3 The change of the Fick diffusivity $D_{14}$ as function of concentration is shown in Eq. A10. This

4 empirical equation was obtained from data on the diffusivity of BSA at isoelectric conditions ( $\mathrm{pH} 4.9)$

5 from the work of Fair et al. and Gaigalas et al. [38, 40]. By combining this equation with Eq. A9 and

$6 \quad$ Eq.23, the final expression for $\bigoplus_{14}^{\mathrm{V}}$ as function of $\varphi_{1}$ can be obtained (Eq. A11). This expression is

7 useful at any $\mathrm{pH}$ and ion strength because the M-S coefficients represent only the friction between

8 components and do not contain non-idealities due to electrical interactions.

$$
D_{14}=D_{14}^{\infty}\left[0.21+0.79 \exp \left(-4.1 \varphi_{1}\right)\right]
$$

9

$$
\bigoplus_{14}^{V}=D_{14}^{\infty} \frac{0.21+0.79 \exp \left(-4.1 \varphi_{1}\right)}{\frac{1+4 \varphi_{1}+4 \varphi_{1}^{2}-4 \varphi_{1}^{3}+\varphi_{1}^{4}}{\left(1-\varphi_{1}\right)^{3}}}
$$

\section{B. Calculation of the osmotic pressure.}

$15 \quad \ln x_{4}+\ln \gamma_{4}=-\frac{\bar{V}_{4}}{R T} \Pi$

Since $x_{4}$ is a number that is very close to 1 , then: $\ln x_{4} \approx x_{4}-1$.

17

18

$\ln \gamma_{4}=-\frac{\bar{V}_{4}}{R T} \Pi-\left(x_{4}-1\right)$
Based on the work of Noordman et al., the osmotic pressure can be calculated by simply considering the concentration of the components of the mixture according to the Donnan distribution [28]. From Eq. 17 we can obtain the relation between osmotic pressure and water activity.

$\ln \gamma_{4}=-\frac{\bar{V}_{4}}{R T} \Pi+x_{1}+x_{2}+x_{3}$ 


$$
\Pi=-\frac{R T}{\bar{V}_{4}}\left(\ln \gamma_{4}-x_{1}-x_{2}-x_{3}\right)
$$

2 Even under concentrated conditions, the total number of moles in the system will be defined mostly by

3 the amount of water in the system, thus $C_{T} \approx \frac{\varphi_{4}}{\bar{V}_{4}}$. Considering this relation and Eq. B1 we can obtain

4 the following relation for the osmotic pressure.

$5 \quad \Pi=-\frac{R T}{\bar{V}_{4}}\left(\ln \gamma_{4}-\frac{\varphi_{1} \bar{V}_{4}}{\bar{V}_{1} \varphi_{4}}-\frac{\varphi_{2} \bar{V}_{4}}{\bar{V}_{2} \varphi_{4}}-\frac{\varphi_{3} \bar{V}_{4}}{\bar{V}_{3} \varphi_{4}}\right)$

$$
\Pi=-\frac{R T}{\varphi_{4}}\left(\frac{\ln \gamma_{4} \varphi_{4}}{\bar{V}_{4}}-\frac{\varphi_{1}}{\bar{V}_{1}}-\frac{\varphi_{2}}{\bar{V}_{2}}-\frac{\varphi_{3}}{\bar{V}_{3}}\right)
$$

6

7 The non-ideality produced by the excluded volume are due to BSA. Therefore, from our 'imaginary'

8 binary system (Eq. 18), an expression for $\ln \gamma_{4}$ (Eq. B3) can be derived in the following way:

$9 \quad \ln a_{4}=-\frac{\overline{V_{4}}}{\bar{V}_{1}}\left(\frac{\varphi_{1}+\varphi_{1}^{2}+\varphi_{1}^{3}-\varphi_{1}^{4}}{\left(1-\varphi_{1}\right)^{3}}\right)$

$10 \quad \ln x_{4}+\ln \gamma_{4}=-\frac{\overline{V_{4}}}{\bar{V}_{1}}\left(\frac{\varphi_{1}+\varphi_{1}^{2}+\varphi_{1}^{3}-\varphi_{1}^{4}}{\left(1-\varphi_{1}\right)^{3}}\right)$

11 In our system $x_{4} \approx 1$, thus $\ln x_{4} \approx x_{4}-1$, thus $\ln x_{4} \approx-x_{1}$. Considering Eq. A5 we obtain:

$12 \frac{\varphi_{1} \bar{V}_{4}}{\bar{V}_{1}\left(1-\varphi_{1}\right)}+\ln \gamma_{4}=-\frac{\overline{V_{4}}}{\bar{V}_{1}}\left(\frac{\varphi_{1}+\varphi_{1}^{2}+\varphi_{1}^{3}-\varphi_{1}^{4}}{\left(1-\varphi_{1}\right)^{3}}\right)$,

which results in a simpler expression:

$$
\ln \gamma_{4}=\frac{\bar{V}_{4}}{\bar{V}_{1}} \frac{\varphi_{1}^{4}-3 \varphi_{1}^{2}}{\left(1-\varphi_{1}\right)^{3}}
$$


1 In the case of the permeate, where no BSA is expected, the system is considered ideal so $\ln \gamma_{4} \approx 0$,

2 and $\varphi_{4} \approx 1$. Consequently Eq. B2 reduces to Van't Hoff equation:

$$
\Pi=-R T\left(-2 C_{2,3}\right)
$$

3 Where $C$ stands for the concentration of the ions in $\mathrm{mol} / \mathrm{m}^{3}$. The factor 2 originates from the fact that

4 the concentration of both ions is the same in the permeate, this value is commonly known as Van't

5 Hoff index.

6

7

8

9

10

11

12

13

14

15

16

17

18

19

20 


\section{REFERENCES}

[1] R. Baker, Membrane Technology and Applications 2nd ed., Wiley, California, USA, 2004.

[2] G. Belfort, R.H. Davis, A.L. Zydney, The behavior of suspensions and macromolecular solutions in crossflow microfiltration, J. Membr. Sci., 96 (1994) 1-58.

[3] W.R. Bowen, J.I. Calvo, A. Hernández, Steps of membrane blocking in flux decline during protein microfiltration, J. Membr. Sci., 101 (1995) 153-165.

[4] S.P. Palecek, A.L. Zydney, Intermolecular electrostatic interactions and their effect on flux and protein deposition during protein filtration, Biotechnol. Progr., 10 (1994) 207-213.

[5] J.G. Wijmans, S. Nakao, C.A. Smolders, Flux limitation in ultrafiltration: Osmotic pressure model and gel layer model, J. Membr. Sci., 20 (1984) 115-124.

[6] M.C. Porter, Concentration polarization with membrane ultrafiltration, Ind. Eng. Chem. Prod. Res. Develop., 11 (1972) 234-248.

[7] R.D. Cohen, R.F. Probstein, Colloidal fouling of reverse osmosis membranes, J. Colloid Interface Sci., 114 (1986) 194-207.

[8] V.L. Vilker, C.K. Colton, K.A. Smith, The osmotic pressure of concentrated protein solutions: Effect of concentration and ph in saline solutions of bovine serum albumin, J. Colloid Interface Sci., 79 (1981) 548-566.

[9] R.H. Davis, J.D. Sherwood, A similarity solution for steady-state crossflow microfiltration, Chem. Eng. Sci., 45 (1990) 3203-3209.

[10] F.W. Altena, G. Belfort, Lateral migration of spherical particles in porous flow channels: application to membrane filtration, Chem. Eng. Sci., 39 (1984) 343-355.

[11] W.R. Bowen, P.M. Williams, The Osmotic Pressure of Electrostatically Stabilized Colloidal Dispersions, J. Colloid Interface Sci., 184 (1996) 241-250.

[12] W.R. Bowen, F. Jenner, Dynamic ultrafiltration model for charged colloidal dispersions: A Wigner-Seitz cell approach, Chem. Eng. Sci., 50 (1995) 1707-1736.

[13] A.-S. Jönsson, B. Jönsson, Ultrafiltration of Colloidal Dispersions-A Theoretical Model of the Concentration Polarization Phenomena, J. Colloid Interface Sci., 180 (1996) 504-518.

[14] P. Bacchin, P. Aimar, R.W. Field, Critical and sustainable fluxes: Theory, experiments and applications, J. Membr. Sci., 281 (2006) 42-69.

[15] P. Bacchin, A possible link between critical and limiting flux for colloidal systems: consideration of critical deposit formation along a membrane, J. Membr. Sci., 228 (2004) 237-241.

[16] A.L. Zydney, Stagnant film model for concentration polarization in membrane systems, J. Membr. Sci., 130 (1997) 275-281.

[17] R. Taylor, R. Krishna, Multicomponent Mass Transfer, John Wiley \& Sons, Inc, New York, 1993.

[18] J.A. Wesselingh, R. Krishna, Mass Transfer in Multicomponent Mixtures, VSSD, Delft, 2000. 
[19] R. Krishna, Describing mixture permeation across polymeric membranes by a combination of Maxwell-Stefan and Flory-Huggins models, Polymer, 103 (2016) 124-131.

[20] C.P. Ribeiro, B.D. Freeman, D.R. Paul, Modeling of multicomponent mass transfer across polymer films using a thermodynamically consistent formulation of the Maxwell-Stefan equations in terms of volume fractions, Polymer, 52 (2011) 3970-3983.

[21] R.F. Probstein, W.-F. Leung, Y. Alliance, Determination of diffusivity and gel concentration in macromolecular solutions by ultrafiltration, The Journal of Physical Chemistry, 83 (1979) 1228-1232. [22] N. El Kadi, N. Taulier, J.Y. Le Huérou, M. Gindre, W. Urbach, I. Nwigwe, P.C. Kahn, M. Waks, Unfolding and Refolding of Bovine Serum Albumin at Acid pH: Ultrasound and Structural Studies, Biophys. J., 91 (2006) 3397-3404.

[23] B. Halle, Protein hydration dynamics in solution: a critical survey, Philosophical Transactions of the Royal Society of London. Series B: Biological Sciences, 359 (2004) 1207-1224.

[24] Prasad S. Sarangapani, Steven D. Hudson, Kalman B. Migler, Jai A. Pathak, The Limitations of an Exclusively Colloidal View of Protein Solution Hydrodynamics and Rheology, Biophys. J., 105 (2013) 2418-2426.

[25] G. Scatchard, I.H. Scheinberg, S.H. Armstrong, Physical Chemistry of Protein Solutions. IV. The Combination of Human Serum Albumin with Chloride Ion1, J. Am. Chem. Soc., 72 (1950) 535-540. [26] C. Tanford, S.A. Swanson, W.S. Shore, Hydrogen Ion Equilibria of Bovine Serum Albumin1, J. Am. Chem. Soc., 77 (1955) 6414-6421.

[27] N.F. Carnahan, K.E. Starling, Equation of State for Nonattracting Rigid Spheres, The Journal of Chemical Physics, 51 (1969) 635-636.

[28] T.R. Noordman, T.H. Ketelaar, F. Donkers, J.A. Wesselingh, Concentration and desalination of protein solutions by ultrafiltration, Chem. Eng. Sci., 57 (2002) 693-703.

[29] M.D. Reboiras, H. Pfister, H. Pauly, Activity coefficients of salts in highly concentrated protein solutions: I. Alkali chlorides in isoionic bovine serum albumin solutions, Biophys. Chem., 9 (1978) $37-46$.

[30] D.G. Leaist, The role of supporting electrolytes in protein diffusion, The Journal of Physical Chemistry, 93 (1989) 474-479.

[31] O. Annunziata, D. Buzatu, J.G. Albright, Protein Diffusiophoresis and Salt Osmotic Diffusion in Aqueous Solutions, The Journal of Physical Chemistry B, 116 (2012) 12694-12705.

[32] J.A. Wesselingh, P. Vonk, Ultrafiltration of a large polyelectrolyte, J. Membr. Sci., 99 (1995) 2127.

[33] P. Walstra, Physical Chemistry of Foods, Marcel Dekker Inc., New York, 2003.

[34] R.S. Faibish, M. Elimelech, Y. Cohen, Effect of Interparticle Electrostatic Double Layer Interactions on Permeate Flux Decline in Crossflow Membrane Filtration of Colloidal Suspensions: An Experimental Investigation, J. Colloid Interface Sci., 204 (1998) 77-86. 
1 [35] C. Ersch, L.L.C. Meijvogel, E. van der Linden, A. Martin, P. Venema, Interactions in protein

2 mixtures. Part I: Second virial coefficients from osmometry, Food Hydrocolloids, 52 (2016) 982-990.

3 [36] J. Wu, J.M. Prausnitz, Osmotic pressures of aqueous bovine serum albumin solutions at high

4 ionic strength, Fluid Phase Equilib., 155 (1999) 139-154.

5 [37] S.P. Palecek, S. Mochizuki, A.L. Zydney, Effect of ionic environment on BSA filtration and the

6 properties of BSA deposits, Desalination, 90 (1993) 147-159.

7 [38] B.D. Fair, D.Y. Chao, A.M. Jamieson, Mutual translational diffusion coefficients in bovine serum

8 albumen solutions measured by quasielastic laser light scattering, J. Colloid Interface Sci., 66 (1978)

$9 \quad 323-330$.

10 [39] G.D.J. Phillies, G.B. Benedek, N.A. Mazer, Diffusion in protein solutions at high concentrations:

11 A study by quasielastic light scattering spectroscopy, The Journal of Chemical Physics, 65 (1976)

$12 \quad 1883-1892$.

13 [40] A.K. Gaigalas, V. Reipa, J.B. Hubbard, J. Edwards, J. Douglas, A non-perturbative relation

14 between the mutual diffusion coefficient, suspension viscosity, and osmotic compressibility: Application to concentrated protein solutions, Chem. Eng. Sci., 50 (1995) 1107-1114. 